\title{
Emotion, Medizin und Volksbelehrung: die Entstehung des «deutschen Kulturfilms»
}

Philipp Osten

\section{Summary}

This article describes the process of professionalisation of medical films in Germany between 1910 and 1920. At the beginning of this decade, government institutions showed a growing interest in hygiene campaigns and started to cooperate with medical experts as well as with professional advertisers. When the German film industry was nationalised at the end of World War I, these informal structures were strengthened. New theories described the film as a most powerful tool for propaganda purposes. This profoundly changed the expectations towards medical films. Now their content had to be bedded into the dramatised form of a photoplay. After 1918, in anticipation of the reprivatisation of the German film industry, government officials of the Weimar Republic developed complex measures to obtain and keep control over a new genre of documentary film which was now called "Deutscher Kulturfilm". Some of the political expectations linked to the Kulturfilm can be exemplified in the first documentary of feature length released by the Berlinbased Universal Film Corporation in 1920. It contained elements of medical films that had been shot during the last decade of the German Empire, and it was newly composed in 1919 to meet the presumable needs of a broader public in an uncertain democratic future.

Keywords: theory of scientific film; Weimar Republic; Deutscher Kulturfilm; Hugo Münsterberg; Erwin Ackerknecht 


\section{Zusammenfassung}

Dieser Artikel schildert die Professionalisierung von Filmen medizinischen Inhalts, die sich in Deutschland innerhalb der zweiten Dekade des 20. Jahrhunderts analog zu einem vermehrten staatlichen Interesse an der hygienischen Volksbelehrung vollzog. Im Zuge der Zentralisierung der deutschen Filmindustrie gegen Ende des Ersten Weltkrieges wurden halbstaatlichen Behörden weitreichende Kompetenzen zugestanden. Neue Theorien würdigten die Suggestionskraft des Films und empfahlen ihn als ideales Propagandainstrument. Gleichzeitig veränderte sich der Anspruch, der an Filme medizinischen Inhalts gestellt wurde. Neben die Vermittlung von Wissen und Fakten trat die dramaturgische Aufbereitung des Stoffes. Nach 1918 versuchten einige staatliche Stellen ihre Oberhoheit über jene Gruppe von Filmen zu behalten, die nun «Kulturfilme» genannt wurden:Während Spielfilmproduktionen mit Hilfe von Zensurbestimmungen nur ex post kontrolliert wurden, entstanden für Kulturfilme subtile und überaus effiziente Massnahmen staatlicher Einflussnahme, die bereits bei der Planung und Entwicklung von Produktionen einsetzten. Am Beispiel einer frühen, als «der erste abendfüllende Kulturfilm der Ufa» bezeichneten Produktion, die in den Jahren 1910 bis 1920 hergestellt wurde, werden die Traditionslinien öffentlicher Gesundheitsaufklärung nachgezeichnet. Dieser Film demonstriert darüber hinaus, wie sich die Verantwortlichen eine konkrete politische Beeinflussung der Bevölkerung durch das neue Medium vorstellten.

\section{Fragestellung}

Im Deutschland der zwanziger Jahre entstanden zahlreiche Dokumentarfilme zu medizinischen Themen. Sie waren überwiegend von guter technischer Qualität, waren anschaulich und legten Wert auf anspruchsvolle Didaktik. Viele von ihnen enthielten Trickfilmelemente. Nicht allein der darzustellende Sachverhalt bestimmte den Inhalt. Schauspieler und fiktive Handlungen rahmten die Aussagen. Gelegentlich kam so etwas wie Humor zum Einsatz, wenn auch in deutlich geringerem Masse als in den Artikeln der Kritiker dieser Filme. Die Themen waren vielseitig: Filme warnten vor Tuberkulose und Geschlechtskrankheiten, Kurpfuscherei und Unsauberkeit, oder sie erläuterten prophylaktische Massnahmen. Einige priesen Therapieverfahren, andere schilderten das Konzept von Heilanstalten oder Fürsorgestellen. Beliebter waren die sogenannten «Steinachfilme», in denen es etwas über die endokrinologischen Grundlagen der Sexualität zu sehen gab. 
Warum aber gleichen sich all diese Filme so sehr? Warum transportieren sie alle so ähnliche Wertvorstellungen? Die Gleichförmigkeit deutscher Dokumentarfilme ist ein so hervorstechendes Merkmal, dass für sie ein eigener Begriff geprägt wurde: Kulturfilm. War der Entwurf der speziellen 〈Gattung〉 Kulturfilme etwa geplant? Das ist schwer vorstellbar in einer Zeit, in der sich Moralvorstellungen so rasant veränderten wie kaum zuvor. Keine Stadt galt in den Zwanzigern als so abwechslungsreich und reizvoll wie Berlin. Warum wurden dort mit so anspruchsvollen Mitteln Filme gedreht, die mit erhobenem Zeigefinger Belehrungen zu eher brisanten Themen in einer Weise verbreiteten, die selbst die offiziellen Zensoren «langweilig» fanden? Wenn Gleichförmigkeit herrscht, wo Vielfalt zu erwarten wäre, müssen zwei Elemente der Beeinflussung gegeben sein: Zum einen muss ein übergeordnetes Konzept existieren; eine Theorie oder eine These, an der sich Filmproduzenten und Drehbuchautoren orientierten. Zum anderen muss die Konformität sanktioniert sein, sei es durch ökonomische Mittel oder durch exekutive Lenkungsmechanismen.

Diese beiden Elemente gilt es herauszuarbeiten. Ich habe meinen Beitrag daher in vier Abschnitte gegliedert. Zunächst schildere ich die Ausgangslage. Medizinfilme waren in den Anfangsjahren vor allem eine Jahrmarktsattraktion. Und doch gab es Bemühungen in zwei Richtungen: Filme wurden für Ausbildungszwecke innerhalb der Medizin hergestellt, und es entstanden Produktionen, die sich an eine breite Öffentlichkeit wandten, ohne ein Spektakel erzeugen zu wollen. Im zweiten Teil untersuche ich zwei theoretische Konzepte. Das eine stammt von Hugo Münsterberg (1863-1916), dessen Filmtheorie unter heutigen Filmfreunden «Kultstatus» geniesst. Dementsprechend häufig wird auf ihn Bezug genommen. Sein Konzept ist die Grundlage des Propagandafilms per se. Weniger bekannt, um nicht zu sagen, in der Filmfanliteratur völlig unbeachtet und in der Fachliteratur höchstens kursorisch erwähnt, sind die Thesen des Breslauer Bibliothekars Erwin Ackerknecht (1880-1960)1. Sein Konzept ist vorsichtiger als das von Münsterberg. Es eignet sich nicht so sehr, Begeisterung zu wecken, aber es schuf die Strukturen, die nötig waren, um Münsterbergs Initialzündung wirksam werden zu lassen. Im dritten Teil frage ich nach den Einflüssen, die die Entstehung der eigenartigen Gattung «deutscher Kulturfilm» ermöglichten. Gab es ein Interesse, nach dem Ersten Weltkrieg die Privatisierung der deutschen Filmindustrie - zumindest für die Dokumentarfilmsparte -

1 Erwin Ackerknecht gilt als Initiator der deutschen Volkshochschulbewegung in den 1920er Jahren. Ab 1947 leitete er das Schiller Nationalmuseum in Marbach. Er war der Vater des Medizinhistorikers Erwin H. Ackerknecht (1906-1988). 
zu verhindern? Ein Gutachten aus der Reichskanzlei legt das nahe. Aber wie konnte so ein Vorhaben am Parlament vorbei verwirklicht werden? Bei der Antwort gilt es weniger, auf die Einführung einer Filmzensur zu achten als auf die neugeschaffenen Strukturen, die bereits bei der Planung eines neuen Films Einfluss auf seine Existenz nahmen. Der vierte Teil besteht in dem Experiment, die zuvor dargelegten Mechanismen anhand der Geschichte eines einzelnen Films deutlich zu machen. Die Wahl fiel auf den 1920 uraufgeführten Film, «Krüppelnot und Krüppelhilfe», der sich als eine Chimäre aus zahlreichen Szenen entpuppte, die über einen Zeitraum von zehn Jahren hinweg gedreht wurden.

\section{Jahrmarkt, Staat und Industrie: die Professionalisierung hygienischer Filme zur Zeit des Kaiserreiches}

Am 3. Januar 1896 stach sich eine Frau aus Berlin Rixdorf beim Waschen einer Gardine eine Nähnadel so unglücklich in die Hand, dass die Spitze abbrach und tief in der Muskulatur des Daumenballens steckenblieb. Wenige Wochen später erschien eine Abbildung ihrer Hand als erste zu diagnostischen Zwecken gefertigte Röntgen-Fotografie in der Deutschen Medizinischen Wochenschrift ${ }^{2}$. An dem Tag, an dem die Verletzung erfolgt war, hatten die ersten Sonderdrucke von Wilhelm Conrad Röntgens «Über eine neue Art von Strahlen» Berlin erreicht ${ }^{3}$. Die Aufnahme des Handskeletts wurde nicht in einem grossen Krankenhaus oder in der Forschungseinrichtung eines physikalischen Instituts angefertigt. Der behandelnde Arzt hatte seine Patientin in den ersten Stock eines Optikergeschäfts in der Friedrichstrasse 95 geführt. Arzt und Optiker exponierten die Hand der Patientin so lange unter der Kathodenstrahlröhre, bis sich auf der fotografischen Platte Skelett und Nadelspitze klar abzeichneten. Die Belichtung der gelungenen Aufnahme hatte schliesslich knapp eine Stunde gedauert.

Gelegen auf halbem Weg zwischen Universitätsinstituten und Charité nahm das Ladengeschäft mit überdimensionalem Fernglas als Zunftschild (Abb.1) eine Schlüsselrolle für die materielle Kultur des wissenschaftlichen Berlin um 1900 ein, zumindest was die Konstruktion optischer Instrumente

2 Biesalski 1896. Ein Überblick der ersten Artikel zur Röntgenstrahlung findet sich in: Journalliteratur 1897/1898. Biesalskis Aufnahme fiel im Kontext der zeitgenössischen Publikationen allein deshalb aus dem Rahmen, weil der Verfasser die Hand der Patientin anhand des Röntgenbildes vermass und die Nadelspitze anschliessend erfolgreich entfernte.

3 Eine Chronologie findet sich bei: Fölsing 1995, 158ff. Parallele Entwicklungen in der Schweiz schildert aus aktueller wissenschaftshistorischer Perspektive: Dommann 2003, 7-20. 


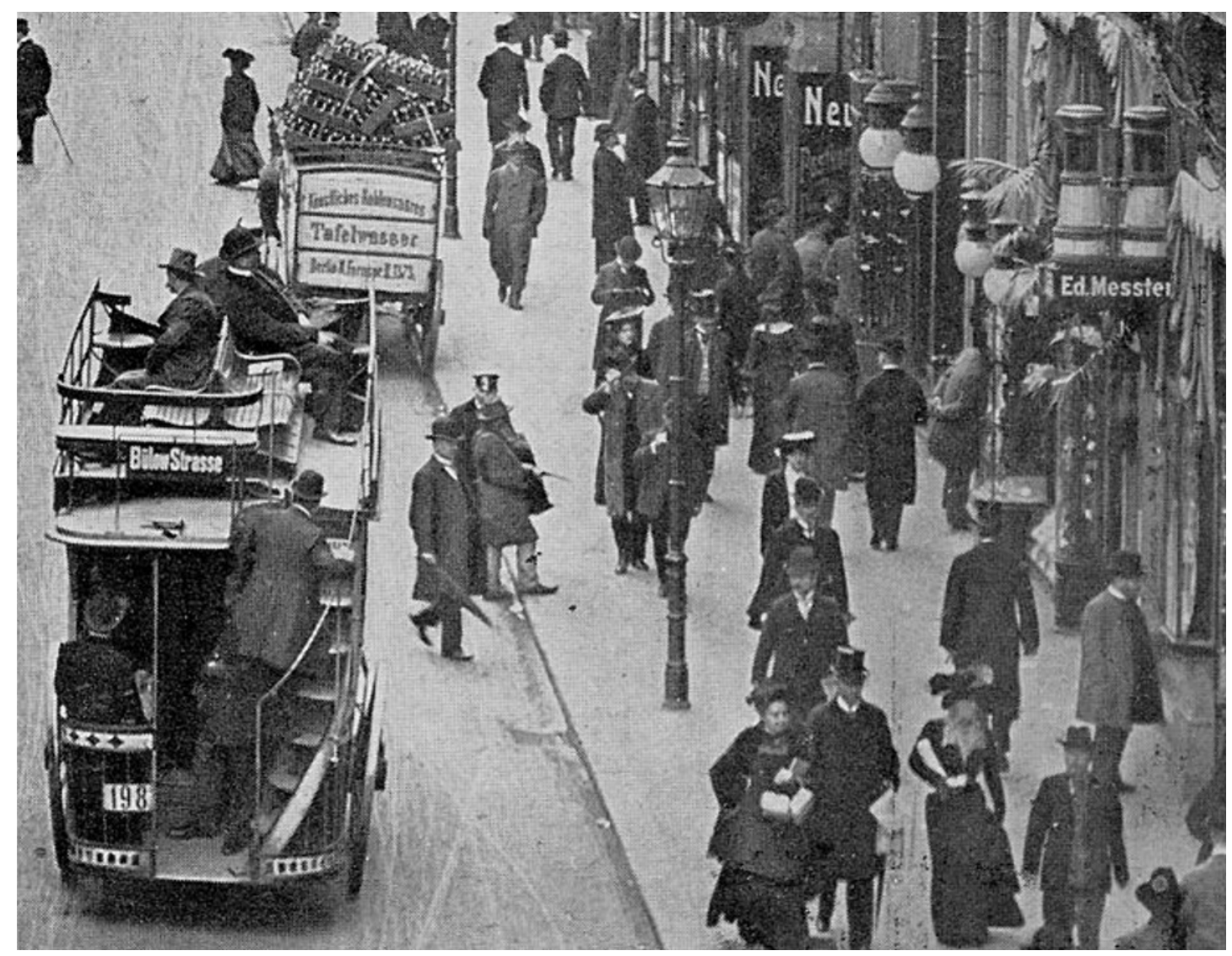

Abb. 1. Blick von einer S-Bahn-Brücke auf das Optikergeschäft Ed. Messter in der Berliner Friedrichstrasse. Fotografie aus: Berliner Leben 1900.

und Aufzeichnungsgeräte anbelangt. Schon bald nach ihrer Gründung im Jahr 1859 war die Firma Ed. Messter durch die Herstellung von Mikroskopen bekannt geworden, während des Siegeszugs der Bakteriologie konnte sie ihren Marktanteil behaupten, das Sortiment wurde dem Bedarf der nahen naturwissenschaftlichen und klinischen Institute angepasst ${ }^{4}$. Bald genoss die Firma den Ruf, nach Vorgaben von Physikern, Pathologen und Zoologen die jeweils neueste Labortechnik individuellen Anforderungen anzupassen. Wenn Anfang des Jahres 1896 irgendwo in Berlin eine für Röntgenversuche geeignete Kathodenstrahlröhre aufzufinden war, dann hier. Neben den Forschern naturwissenschaftlicher Institute suchten auch Betreiber von Varietés, beleuchteten Springbrunnen und Panoptiken feinmechanischen Rat in Messters Optikerladen. Die Wartung von Kaiserpanoramen war hier eine ähnlich alltägliche Dienstleistung wie die Röntgendiagnostik. Und so brachte - ebenfalls im

4 Preisverzeichnis sämtlicher optischer, mechanischer und chirurgischer Instrumente. Nachlass Oskar Messter. Bundesarchiv Berlin. 
Jahr 1896 - ein am Varieté Wintergarten unter Vertrag stehender Schausteller den funktionsunfähigen Prototyp eines Filmvorführgerätes vorbei, den Messter für ihn in Gang setzen sollte ${ }^{5}$. Bald galt Oskar Messter (1866-1943), der Sohn des Firmengründers, in Deutschland als Erfinder des Filmprojektors. Ein Ruhm, der dem später erfolgreichsten Filmproduzenten der Kaiserzeit mehr aus nationalem Pathos zufiel, denn zuverlässige Kameras und Projektoren setzte Messter - wie seine Mikroskope - aus allgemein erhältlichen Bauteilen zusammen ${ }^{6}$. Drei Monate nach der Nadel-Fotografie waren Messter in demselben Zimmer, in dem er auch seine Röntgenexperimente durchführte, erste Filmaufnahmen geglückt. Sie dokumentieren den Zugverkehr auf jener S-Bahn-Brücke, von der aus das Foto (Abb.1) aufgenommen wurde. Der abgedunkelte Raum im ersten Stock des Optikerladens im nördlichen Drittel der Friedrichstrasse symbolisiert recht anschaulich die enge Verbindung zwischen Wissenschaft, Jahrmarkt und Industrie in der Frühphase des Films.

In welchen, gelegentlich auch zwielichtigen Kontexten wissenschaftliche Filme in der Zeit vor dem Ersten Weltkrieg aufgeführt wurden, ist nicht einfach zu ermitteln. Dem Film haftete keine wissenschaftliche Aura an, wie etwa Etienne-Jules Mareys (1830-1904) und Eadweard Muybridges (1830-1904) Serienaufnahmen von Bewegungsabläufen. Und auch die an der Salpêtrière angefertigten Abbildungen von Hysterikerinnen, die wir als Einzelbilder kennen, wurden mit einer neunäugigen Plattenkamera aufgenommen, deren elektromagnetische Verschlussmechanismen in enger zeitlicher Folge nacheinander ausgelöst wurden ${ }^{7}$. Die Zerlegung eines Bewegungsablaufes in seine Elemente besass analytische Qualität, die Zusammensetzung schneller Bildfolgen zur Illusion der Bewegung hingegen erinnerte an einen Taschenspielertrick. Wissenschaftlich anerkannt wurden in der Zeit vor dem Ersten Weltkrieg nur solche Filme, die der Forschung dienten. Lobend hob der Dokumentarfilm-Chronist Ernst Degener Arbeiten hervor, die die Kontraktion des Herzens oder pathologische Gangbilder erklärten $^{8}$. Der Zeitraffer wurde zum Takt der frühen Mikrokinematographie: Ob im Dunkelfeld zitternde Treponemen, Zellteilungen oder die Verpuppung einer Raupe zum Schmetterling - das neue Wiedergabemedium

\footnotetext{
5 Koerber 1994.

6 Messter verfasste in den dreissiger Jahren eine Autobiographie, in der er seine Pionierleistungen als Erfinder herausstellte, vgl. Messter 1936. Zum Teil korrigiert jüngere Forschungsliteratur dieses Bild; sie hebt die unternehmerische Komponente hervor: Loiperdinger/Pulch 1998; Karlsch 1994.

7 Gunthert 2000.

8 Degener 1930.
} 
animierte Bilderfolgen. Die Zerlegung eines Ablaufes in Einzelbilder, das Prinzip der Aufzeichnungen von Muybridge und Marey, das in einer Definition einzelner Stadien eines fliessenden Vorgangs bestand, kehrte sich in der willkürlichen chronologischen Neuanordnung des Zeitraffers um. Der Literaturwissenschaftler Erwin Ackerknecht fasste diesen Eindruck 1918 mit knappen Worten zusammen: «Das Laufbild wirkt synthetisch, das Stehbild analytisch.» ${ }^{9}$

Bereits der früheste heute bekannte Film medizinischen Inhalts, der in Deutschland gedreht wurde, trug dazu bei, das neue Medium unter wissenschaftlichen Gesichtspunkten in Verruf zu bringen: In Berlin liess sich 1898 der Leiter der Chirurgischen Universitätsklinik von Oskar Messter bei einer Amputation aufnehmen ${ }^{10}$. Die Technik dieser Operation vereinte alle Vorzüge eines erfolgreichen Jahrmarktfilms. Der gesamte Eingriff dauert eine Minute und 15 Sekunden. Um die bei Knochen und Gelenken besonders hohe Infektionsgefahr zu minimieren, wetteiferten Chirurgen, wer der schnellste Operateur sei. Messter hatte seine Kamera auf einem Stativ befestigt. Aus unveränderter Position verfolgten die Zuschauer, wie Ernst von Bergmann (1836-1907), unterstützt von zwei Krankenschwestern und drei Assistenten, mit Skalpell und Säge einen Unterschenkel amputiert. Mit einer hastigen Verbeugung, ausgeführt unmittelbar nach dem letzten Handgriff am Patienten, deutete Bergmann dem Kameramann an, er könne die Aufnahme beenden. Das Echo der Fachkollegen war verheerend. Zum einen war das Operationsfeld kaum zu sehen, was den Einsatz als Lehrfilm ad absurdum führte; schlimmer noch, man warf dem angesehenen Ordinarius vor, er geriere sich wie ein Schausteller. In den folgenden Jahren sollte Bergmann aus Jahrmarktbuden und Varietésälen tausendfach Applaus für seine Darbietung erhalten, der immer dann einsetzte, als er auf der Leinwand seine Verbeugung vollführte ${ }^{11}$. Seit 1942 kann der Film über das Göttinger Institut für den wissenschaftlichen Film bezogen werden und wird bis heute gerne im Rahmenprogramm von Chirurgie-Kongressen gezeigt.

Didaktische Filme nahmen vor dem Beginn des Ersten Weltkrieges weniger im universitären Unterricht als vielmehr in der Produktwerbung einen festen Platz ein. Das visuelle Massenmedium dieser Zeit waren Ausstellungen. Industrielle Leistungsschauen zogen mitunter mehrere hunderttausend Besucher an. Glaubt man dem Berliner Tageblatt, so war das Publikum von

9 Ackerknecht 1928,11-67; Ackerknecht 1918, 27.

10 Angaben über Jahr und Produzent der Aufnahme divergieren. Vgl. Haase 1941.

11 Ein Schicksal, das der Film von 1898 mit vielen anderen Operationsfilmen teilte. Vgl. Rothe 1924. 
einer filmischen Inszenierung auf der Ausstellung 〈Das Gas〉 in den frühen 1910er Jahren besonders beeindruckt ${ }^{12}$. Eine Firma verzichtete in ihrer als Zentralbau errichteten Halle völlig auf die Ausstellung von Produkten. Stattdessen projizierten mehrere Vorführgeräte Filme an die Aussenwände einer Rotunde. Sie präsentierten die technische Entwicklung ihrer Geräte, die Herstellung in den Fabriken und den Gebrauch durch glückliche Endverbraucher. In regelmässigen Abständen flammte oben in der Kuppel über den Köpfen der Besucher ein Leuchtfeuer in 120000 Kerzenstärken auf ${ }^{13}$. Das Jahrmarktdiorama erfuhr in dieser Inszenierung aus Film und Flutlicht einen neuen, multimedialen Höhepunkt.

Einem grösseren Publikum wurden Filme medizinischen Inhalts auf der Dresdner Hygiene-Ausstellung von 1911 vorgeführt. Mit acht Millionen Besuchern gilt sie als die erfolgreichste Massenveranstaltung der Kaiserzeit. In Kooperation mit staatlichen Stellen wurden hierfür Konzepte erarbeitet, die in den folgenden 20 Jahren die Grundlage der Hygienischen Volksbelehrung in Deutschland bildeten ${ }^{14}$. Dem hier vorexerzierten Zusammenwirken von Staat, Firmen und Institutionen, das im Folgenden beschrieben wird, sollte später bei der Entwicklung des Kulturfilms eine besondere Bedeutung zukommen. Ihren Gewinn erwirtschaftete die Ausstellung auf ähnliche Weise wie herkömmliche Messen. Industrieunternehmen konnten Kojen buchen oder ganze Gebäude errichten, in denen sie ihre Produkte präsentierten, ergänzt durch ein breites gastronomisches und vergnügungsrelevantes Angebot, das an Pächter mit eigenen Konzepten ausgeschrieben wurde ${ }^{15}$. Hygiene diente in Dresden nicht allein als rahmendes Motto. Der Veranstalter, der durch das Mundwasser Odol bekannte Fabrikant Karl August Lingner (1861-1916), lud internationale Organisationen, karitative Institutionen und öffentliche Einrichtungen ein, die Ziele ihrer wissenschaftlichen oder gemeinnützigen Arbeit dem Publikum in ansprechender Form vorzuführen. Die Gestaltung dieses Rahmenprogramms übernahm eine Auswahlkommission in enger Kooperation mit Ministerien des Reiches und des preussischen und sächsischen Staates. So wurde beispielsweise die Abteilung Krankenfürsorge, für die ein eigenes Gebäude errichtet worden war, von dem Leiter der Medizinalabteilung des preussischen Ministeriums der geistlichen Unterrichts- und Medizinalangelegenheiten, Eduard Dietrich (1860-

12 Albrecht 1914.

13 Kerzenstärke war die um die Jahrhundertwende übliche Masseinheit für natürliches Licht, sie wurde in den 1950er Jahren durch die fotoelektrisch definierte SI-Einheit Candela ersetzt.

14 Vergleiche Osten 2005.

15 Vorstand des Vereins zur Veranstaltung der Internationalen Hygiene-Ausstellung Dresden 1911 e.V. 1909, S. 2. 
1947), inhaltlich verantwortet ${ }^{16}$. Damit im Gegensatz zu den gekonnten Inszenierungen der Industrieartikel die sogenannten wissenschaftlichen Abteilungen ihr Publikum nicht zu sehr langweilten oder mit der Darstellung von Krankheiten abschreckten, wurde ein «Statistisches Büro der HygieneAusstellung» eingerichtet. Es sollte die Aussteller in Hinblick auf die Gestaltung der Kojen, in der grafischen Aufbereitung von Schaubildern und bei der Produktion kurzer Filme beraten und eine möglichst einheitliche Präsentation gewährleisten ${ }^{17}$.

So wurde in einem Film das Leben körperbehinderter Kinder nicht als «Elend», sondern als «Jubel, Frohsinn und alles Glück der Kindheit» dargestellt: «Alles Unangenehme und Abstossende ist vermieden, es kommt lediglich das Versöhnliche und Heitere [...] zur Anschauung und übt nach der bisherigen Erfahrung auf Laien eine ungewöhnlich aufklärende, werbende, ja begeisternde Wirkung aus» ${ }^{18}$, berichtete die halb-staatliche Deutsche Vereinigung für Krüppelfürsorge in ihrem Publikationsorgan über einen auf ihre Rechnung für die Dresdner Ausstellung produzierten Film. Das für derartige Vorgaben verantwortliche Statistische Büro der Dresdner Hygiene-Ausstellung erfuhr in den folgenden Jahren eine beachtliche Karriere. Nach dem Ende der Messe vertrieb es sämtliche unter seiner Beratung hergestellten Schaubilder als Diaserien, es diente als Filmverleih und beriet Schulärzte, Stiftungen und Lehrer in der Darbietung von Lichtbildvorträgen und Stummfilmvorführungen. Mit dem Beginn des Ersten Weltkrieges war es dieses Statistische Büro, das in enger Abstimmung mit dem Kriegsministerium die Bestückung der grossen Kriegsausstellungen kuratierte. Finanziert wurden diese staatlichen Propagandaschauen, wie schon die Hygieneausstellungen, durch die Gebühren aus der Zurschaustellung industrieller Produkte.

Unter der Ägide des Ministerialbeamten Dietrich stand dem Statistischen Büro seit der Dresdner Hygiene-Ausstellung für inhaltliche Fragen ein

16 Der geheime Medizinalrat Eduard Dietrich begann seine Karriere als Kreisphysikus von Liebenwerda. 1900 trat er in die Medizinalabteilung des preussischen Ministeriums der geistlichen Unterrichts- und Medizinalangelegenheiten [im folgenden Text kurz: Kultusministerium] ein und konzipierte das im selben Jahr verabschiedete Hebammengesetz. Reformen der Krankenpflegeausbildung und weiterer Berufsordnungen medizinischer Hilfsberufe gehen massgeblich auf ihn zurück. Viele Gründungen privat finanzierter Wohltätigkeitsstiftungen auf dem Gebiet der Säuglings- und Jugendfürsorge, die Gelder für zahlreiche Krankenhausbauten bereitstellten, sind der Initiative seiner Abteilung zu verdanken. Im Jahr 1923 wurde er Leiter der von ihm aufgebauten Abteilung Volkswohlfahrt im preussischen Wohlfahrtsministerium. Auf dem Gebiet des Medizinalwesens steht Dietrich wie kaum ein Zweiter für die personelle und inhaltliche Kontinuität des preussischen Beamtentums über das Ende des Kaiserreiches hinaus. Zu Dietrich vgl. Lentz 1930.

17 Roesle 1911, 19.

18 Zum Zwecke der Propaganda 1912. 
Beraterstab aus herausragenden Vertretern der wichtigsten medizinischen Fächer zur Verfügung. Als es nach der Novemberrevolution zu einer Neuordnung der Medizinalabteilung des preussischen Wohlfahrtsministeriums kam, entwickelte sich aus diesem Gremium der mit weitgehenden Beraterkompetenzen ausgestattete (Preussische Landesgesundheitsrat), dem Eduard Dietrich bis 1926 vorstand. Ab 1922 erhielt darüber hinaus die personell sehr ähnlich besetzte «Reichsarbeitsgemeinschaft für soziale Hygiene und Demographie> Bedeutung für Weichenstellungen der Gesundheitspolitik in der Weimarer Republik; sie verfolgte ein Programm, das vor allem an sozial- und rassenhygienischen Maximen orientiert war ${ }^{19}$. Diese Expertengremien sicherten politische Kontinuität über das Ende des Kaiserreiches hinweg. Nach gleichem Muster (und in zum Teil identischer personeller $\mathrm{Zu}$ sammensetzung) sollte in den Anfangsjahren der Weimarer Republik ein nicht durch politisches Mandat, sondern durch Expertentum legitimierter Ausschuss darüber entscheiden, welchem Film der Status 〈Kulturfilm〉 zugebilligt wurde und welchem nicht.

\section{Die Konzepte von Hugo Münsterberg und Erwin Ackerknecht und ihre Bedeutung für das Erwachen eines staatlichen Interesses am Film im Ersten Weltkrieg}

Bei dem zunehmenden Raum, den Filme auf Messen, Ausstellungen und in der Werbung einnahmen, wundert es kaum, dass auch die früheste wissenschaftliche Studie über die psychologische Wirkung des Films aus dem Bereich der Wirtschaft stammt: von dem Begründer der sogenannten Industriellen Psychotechnik, Hugo Münsterberg ${ }^{20}$. Münsterberg studierte in Leipzig, Genf und Heidelberg Medizin und Philosophie. Er promovierte an beiden Fakultäten ${ }^{21}$. Nach seiner Habilitation in Freiburg im Breisgau wurde ihm zunächst, möglicherweise auch aus antisemitischen Ressentiments ${ }^{22}$, ein Ruf verwehrt. Die Harvard Universität richtete ihm 1892 ein Laboratorium für experimentelle Psychologie ein, 1895 schuf sie ihm einen eigenen Lehrstuhl. Eignungsprüfungen für Industriearbeiter und die Perfektionierung von Produktionsabläufen machten ihn zu einem gefragten Experten. Sein von William Stern und Wilhelm Wundt beeinflusster Ansatz berücksichtigte

19 Vergleiche Reyer 1991; Saretzki 2000, 506.

20 Eine gelungene wissenschaftshistorische Einordnung der Psychotechnik Hugo Münsterbergs findet sich bei: Vöhringer 2007, 25-34 und 81-90.

21 Keller 1979, 7-118.

22 Lück 1997. 
vor allem sinnesphysiologische Aspekte, psychoanalytische Theorien lehnte er ab.

Münsterberg wurde US-amerikanischer Staatsbürger, einen Ruf nach Königsberg lehnte er $1905 \mathrm{ab}^{23}$. Als einen Höhepunkt seiner beruflichen Laufbahn betrachtete er seine Austauschprofessur an der Berliner Universität im Wintersemester 1910/11, in deren Verlauf er unter anderem Gründungsdirektor des Amerika-Institutes der Staatsbibliothek wurde und eine Vorlesung in Anwesenheit des Kaisers hielt ${ }^{24}$. Bald darauf versah ihn Wilhelm II. mit dem Roten Adlerorden ${ }^{25}$. Münsterbergs deutsch-amerikanischer Patriotismus wurde in den USA sprichwörtlich ${ }^{26}$. Kurz nach Beginn des Krieges bezeichnete die britische Times Münsterberg als einen Agenten des Kaisers:

On all occasions, when German-American questions are in the air he deserts his psychological laboratory at Cambridge and becomes a fervid protagonist of the German cause. ${ }^{27}$

Münsterbergs Interesse an Filmen setzt mit dem Beginn des Ersten Weltkrieges ein. Die Bedeutung des Mediums als Bildungsinstrument schätzte er kulturpessimistisch ein:

The power of the moving pictures to supplement the school room and the newspaper and the library by spreading information and knowledge is, after all, secondary to their general task, to bring entertainment and amusement to the masses. ${ }^{28}$

Das minderte keineswegs die Bedeutung, die der Psychologe dem Film in seinem 1916 erschienenen Buch «The Photoplay» zuschrieb. Münsterberg arbeitete die Besonderheiten des Films nicht aus der Sicht seiner Produzenten, sondern aus der Perspektive des Zuschauers heraus. In einem Lichtspielhaus befinde sich das gebannte Publikum in einem Zustand erhöhter Beeinflussbarkeit, hier sei es besonders aufnahmefähig für Suggestionen. Suggestionen bestanden für Münsterberg aus demselben psychologischen Material wie Imagination oder Erinnerung. In Schnittfolge, Wahl des Bildausschnittes und Kameraführung werde das Sehen des Zuschauers geleitet; ganz so, als wenn er selbst seine Aufmerksamkeit einem bestimmten Gegen-

23 «Declines German Chair. Prof. Hugo Muensterberg Decides to Remain with Harvard», The Washington Post (April 14, 1905).

24 Münsterberg, Hugo, «A Contradiction of the Allegations that the Kaiser Was Cool to Him», The New York Times (December 8, 1910) 12.

25 «Decorates Münsterberg», New York Times (August 24,1911).

26 An politischer Literatur erschienen in den USA innerhalb der ersten beiden Jahre nach Kriegsbeginn aus Münsterbergs Feder im Appelton Verlag: The War and America (1914); The Peace and America (1915); Tomorrow. Letters to a Friend in Germany (1916). In Deutschland erschienen: Die Amerikaner (2 Bände) 1914; Amerika und der Weltkrieg. Ein amerikanisches Kriegstagebuch (1915).

27 «From an American Correspondent: the Kaiser's American Agents. The Failure of a Vast Campaign», The Times (September 26, 1914).

28 Münsterberg, Photoplay, 1916, 22. 
stand zuwende. Mit Rückblenden könne Erinnerung imitiert werden. Damit nutze der Film Möglichkeiten, die nur das Unbewusste besitze. Er erzeuge auf unmittelbare Weise Emotion. Zwar müsse ein Film die Realität ein Stück weit abbilden. Aber wertvoll werde er erst dann, wenn er das Kunststück vollbringe, das Gegenständliche zu überwinden, und eine neue Welt schaffe ${ }^{29}$. An dieser Stelle flicht Münsterberg seine Analogie von Wissenschaft und Film ein: Ein Wissenschaftler untersuche ein spezielles Objekt nicht seiner selbst willen, sondern um sich an ihm einen universellen Prozess zu vergegenwärtigen. Genau eine solche Übertragung vom Objekt zum Prinzip müsse der Film bei seinen Betrachtern generieren ${ }^{30}$. Damit entwirft Münsterberg das stilbildende Element des deutschen Kulturfilms ab 1917: Die Handlung eines Films wird zum Gleichnis. Sein Ziel ist es, beim Zuschauer eine höhere Einsicht zu induzieren. Zu erreichen sei dieses Ziel am besten unter dem suggestiven Einfluss eines geleiteten Blicks bei gesteigerter Emotionalität.

In den USA wurde «The Photoplay» mit wenig Sympathie, aber dennoch mit wachem Interesse aufgenommen. Ein Kritiker der New York Times fasste die Kernaussage humorvoll zusammen: Münsterberg erkläre in seinem «windigen und von Redundanzen geprägten Buch», wie sich die im Film gezeigte Aussenwelt im Kopf der Zuschauer in einen gedanklichen Prozess verwandele. Der Film selbst übernehme die Regie über Aufmerksamkeit, Erinnerung und Assoziationen, so dass durchaus nicht gewährleistet sei, ob der Betrachter selbst noch einen Einfluss auf seine eigene Wahrnehmung besitze ${ }^{31}$. Bei aller Ironie, dass Münsterbergs Erkenntnisse für kriegswichtig gehalten wurden, steht ausser Zweifel. Der ehemalige US-Präsident Theodore Roosevelt (1858-1919) attackierte den Psychologen während einer Ansprache im Oktober 1916 mit «den wohl kräftigsten Ausdrücken, die er je gebraucht hat», wie die kirchliche Tageszeitung Christian Science Monitor indigniert feststellte. Die Kraftausdrücke selbst wurden nicht zitiert, wohl aber Roosevelts Unbehagen darüber, dass der Harvard-Professor sich in den Dienst Deutschlands stelle, «ohne sich darum zu scheren, ob seine Handlungen Amerika Nachteile und Schaden zufügen» ${ }^{32}$.

29 Friedrich Kittler räumt Münsterbergs Thesen eine Schlüsselstellung in seiner Theorie von der fortschreitenden medialen Simulation der Welt ein. Vgl. Kittler 1986, 177-269. Die jüngere filmwissenschaftliche Literatur hebt Münsterbergs Verbindung von Unterbewusstsein, Psychotechnik und Suggestion hervor, vgl. Krause/Pethes 2007; Holl 2002, 244 f.

30 Münsterberg, Photoplay, 1916, 153.

31 «Notable Books in Brief Reviews. Professor Muensterberg and Vachel Lindsay in Appreciations of the Art of the Cinema», The New York Times (June 4,1916).

32 «Col. Roosevelt Makes Attacks on Muensterberg», Christian Science Monitor (October 27, 1916). 


\section{Noch schärfer formulierte die New York Times:}

As an officer in the field reports to MACKENSEN or as MACKENSEN reports to HiNDENBURG on his military operations, as an officer of the secret service reports to his chief on his activities in the territory assigned to him, as a diplomatic agent reports to the foreign office, so Dr. MuensterberG reports his work for Germany's ultimate success in the department where he labours. ${ }^{33}$

Vehement wurde auf den Titelseiten der Tageszeitungen diskutiert, ob die Harvard Universität einen Hochschullehrer, der sich selbst als in deutschen Diensten stehend betrachtete, nicht suspendieren müsse ${ }^{34}$. Ihren Höhepunkt erreichte die Auseinandersetzung, als Münsterberg in einer Kirche der von deutschen Einwohnern geprägten Stadt Hoboken (New Jersey) erklärte, deutsche Kultur werde die Welt erobern. «Earth to be Germanized»stand am nächsten Morgen in der Zeitung ${ }^{35}$. Der Psychologe definierte in seiner Rede deutsche Kultur als «the consciousness of nationalism, the subordination of the individual to the national idea» ${ }^{36}$. Wenige Wochen später, am 16. Dezember 1916, starb Hugo Münsterberg mitten in seiner Psychologie-Vorlesung an einem Hirnschlag ${ }^{37}$. Bedingungsloser Patriotismus bei Deutsch-Amerikanern vor Beginn des Ersten Weltkrieges erhielt in den Gazetten einen neuen Namen: Muensterbergism ${ }^{38}$.

Münsterbergs Buch «The Photoplay» rehabilitierte filmische Massenunterhaltung. Film galt für ihn als ein wirkungsvolles Instrument zur Beeinflussung des Publikums. Aber nicht nur das, Münsterberg hatte auch ein Umdenken bei der Bewertung von Filmen hinsichtlich der Frage angeregt,

33 «A Report from the Department of Peace», The New York Times (October 11, 1916). [Hervorhebung durch Kapitälchen im Original.]

34 «Retention of Muensterberg Is Protested. American Rights League Says his Attitudes on War Inconsistent with Duties as Instructor in United States University», Christian Science Monitor (October 21, 1916). Sowie: «Suggest That Harvard Oust Muensterberg. Boston Members of American Rights League Petition the University Authorities», The New York Times (October 22,1916). Sowie: «Muensterberg Case Said to Be Before Harvard. President and Fellows Hold a Meeting but Give No Official Statement», Christian Science Monitor (October 30, 1916). Schon 1915 hatte Münsterberg selbst eine solche Debatte angeheizt, indem er pro forma seinen Rücktritt erklärte, nachdem ein potentieller Erblasser seiner Fakultät geschrieben hatte, er werde sein Testament, in dem er bisher die Harvard Universität mit 10 Millionen Dollar berücksichtige, ändern, falls Münsterberg nicht entlassen werde. Damals wurde der Rücktritt abgelehnt. Vgl.: A. P. Night Wire, «Muensterberg Resigns as Harvard Professor», Los Angeles Times (October 15, 1914).

35 «Muensterberg Sees Kultur Worldwide», The New York Times (October 27, 1916).

36 Ebendort (wie Fussnote 35). Das identische Zitat findet sich auch in: «Prof. Münsterberg Speaks. Hoboken Audience Is Told of Future of German 〈Kultur»», Christian Science Monitor (October 27, 1916). Münsterberg sah sich falsch zitiert und verwies auf sein eben erschienenes Buch «Tomorrow». Vgl. Münsterberg, Hugo, «To the Editor of the New York Times», The New York Times (November 6,1916).

37 «Muensterberg Dies Addressing Class. Harvard Psychologist Falls Unconscious Before Sixty Radcliff Girls», The New York Times (December 17, 1916).

38 Lück 1997. Zur Biographie Münsterbergs vgl. Münsterberg 1922. 
ob es sich bei ihnen um Kulturerzeugnisse handle oder nicht. Der Psychologe hatte das Medium in die Nähe von Musik und Kunst gerückt. Nicht Handlung, Szenenfolge oder Dialoge selbst, sondern per Emotionen übertragene Einsichten und Nachwirkungen bestimmten seine Qualität. Film war im Idealfall eine unmittelbar wirksame Allegorie in laufenden Bildern. Diese neue Sicht des Psychotechnikers Münsterberg auf das Medium Film machte sich die Oberste Heeresleitung in Berlin zu eigen. Am 30. Januar 1917 wurde auf ihr Betreiben ein Bild- und Filmamt (Bufa) im Auswärtigen Amt gegründet. Dieser Schritt leitete die Verstaatlichung des überwiegenden Teils der deutschen Filmindustrie ein, die im Dezember desselben Jahres unter dem Dach der Universum Film A. G. (Ufa) abgeschlossen wurde. Ein illegitimer Vorgang, wie Gutachten, die in Akten der Reichskanzlei erhalten sind, zu Beginn der Weimarer Republik rückblickend feststellten ${ }^{39}$. Die Summe zum Kauf der Produktionsfirmen war nicht durch das Parlament bewilligt worden, stattdessen hatte die Oberste Heeresleitung klandestin Sicherheiten bei der Industrie (darunter bei AEG, Dresdner Bank, Robert Bosch AG, Hamburg-Amerika-Linie und Norddeutschem Lloyd) eingeworben ${ }^{40}$.

Das Gründungsdokument der Ufa enthält den häufig zitierten Satz Ludendorffs: «Der Krieg hat die überragende Macht des Bildes und Films als Aufklärungs- und Beeinflussungsmittel gezeigt.» ${ }^{41}$ Diese Aussage klingt wie ein Echo auf Münsterbergs Theorien. Populär fasste Waldemar Schweisheimer Münsterbergs Regeln von der emotionalen Suggestion des Films im Jahr 1920 in seiner Abhandlung «Die Bedeutung des Films für Soziale Hygiene und Medizin» so zusammen: «Das Filmdrama ist die einzige eine Dauerwirkung erzielende Form des Volksbelehrungsfilms.» Wissenschaftlich interessante Bilder möge ein Durchschnittszuschauer interessiert verfolgen, wenn er sie aber nicht «seelisch verarbeitet, so ist der mit der Vorführung des Films erzielte Nutzen gleich Null» ${ }^{42}$. Ambitionierter drückte es der oberste Kulturfilmwächter der Weimarer Republik, der Pädagoge Felix Lampe (1868-1946), aus:

Weil ins Gefühlsleben die stärkste Wurzel des Wollens hinabtaucht und weil Vorstellungen, Gedächtnis, Phantasie durch das Gefühl stark beeinflusst werden, bedeutet der Bildstreifen wegen seiner Gewalt über Gefühlserregung und Gefühlsablauf eine schneidende Waffe in der Hand des Volkserziehers ${ }^{43}$.

39 Akten betreffend Film-Angelegenheiten. Vom 27. Mai 1919 bis zum 20. Oktober 1920. Bundesarchiv Berlin, Bestand R43 I, 2497.

40 Jung/Mühl-Benninghaus 2006.

41 Schreiben Erich Ludendorffs an das preussische Kriegsministerium vom 4. Juli 1917. Zitiert nach: Barkhausen 1982, 259.

42 Schweisheimer 1920, 13.

43 Lampe 1924. 
Die anfängliche Scheu der Wissenschaftler und Pädagogen vor dem Film ist in dem ab 1917 neu verbreiteten Begriff 〈Kulturfilm〉 festgeschrieben. Noch zu Beginn des Jahrhunderts wäre dies eine contradictio in adjecto gewesen, Kultur und Film waren Gegensätze. Münsterbergs Theorien über die suggestive Wirkung von Film bewirkten ein Umdenken. So erklärt sich, dass auch Künstler in die Filmpropaganda der Obersten Heeresleitung einbezogen wurden ${ }^{44}$. Kaum eine der ambitionierten Produktionen des Bild- und Filmamtes der Obersten Heeresleitung, des Auswärtigen Amtes oder später der Ufa erreichte ihr Publikum noch zu Kriegszeiten. Aus ökonomischen Gründen kamen nur wenige staatliche Kulturfilmproduktionen der Jahre 1917/18 überhaupt zu einem Abschluss ${ }^{45}$. Umso geplanter erscheint der Einsatz von Volksbelehrungsfilmen in den Anfangsjahren der Weimarer Republik. Manche von ihnen lassen sich nur anhand der Angabe ihrer Uraufführung in die Nachkriegszeit datieren. Ein Ufa-Kulturfilm mit dem Titel «Die Folgen der alliierten Hungerblockade» wurde im Mai 1919 vom Auswärtigen Amt mit 5000 Mark finanziert ${ }^{46}$. Ebenso hätten auch zahlreiche Aufklärungsfilme zu Syphilis, Gonorrhoe und zur sogenannten Kriegskrüppelfürsorge gut vor Kriegsende in das Programm von Bufa und Ufa gepasst. Medizinische Volksbelehrungsfilme waren immer auch sozialpolitische Stellungnahmen ${ }^{47}$.

Weitgehend in Vergessenheit geriet, dass noch vor der Gründung der Ufa in den letzten Jahren des Ersten Weltkrieges eine andere Organisation wissenschaftliche Filme sammelte, um sie für Bildung, Unterricht und Propaganda zur Verfügung zu stellen: der «Bilder-Bühnen-Bund Deutscher Städte ${ }^{48}$. Treibender Kopf war der Leiter der Stettiner Stadtbücherei, Erwin Ackerknecht. Ziel des von ihm initiierten Bunds war es, auf kommunaler Ebene ein Netzwerk zur Konzeption, Herstellung und Nutzung von «belehrenden Laufbildern» aufzubauen ${ }^{49}$. Schon in der Vorkriegszeit hatte Ackerknecht ein kommunales «Musterkino» entworfen, um Städten und Gemeinden die Vorführung von Filmen zu erleichtern ${ }^{50}$. Zunächst erhielt er dabei

44 Spuren davon hat der Berliner Filmhistoriker Jeanpaul Goergen anhand von Archivmaterial rekonstruiert. So wurde der spätere Agit-Prop-Künstler John Heartfield (1891-1968) gemeinsam mit dem späteren DADAisten George Grosz (1893-1959) von der Obersten Heeresleitung beauftragt, Trickfilme zu Propagandazwecken anzufertigen. Vgl. Goergen 1994.

45 Die bisher ausführlichste Filmographie deutscher Dokumentarfilme findet sich bei Jung/ Loiperdinger 2006 und Goergen 2005.

46 Auszug aus dem Protokoll der Sitzung des Reichsministeriums vom 27. Mai 1919. Akten betreffend Film-Angelegenheiten, Alte Reichskanzlei. Bundesarchiv Berlin, Bestand R 43 I/ 2497 , Blatt 4.

47 Vergleiche Schmidt, Blick auf den Körper 2000a; Schmidt, Sozialhygienische Filme 2000b.

48 Zum Bilder-Bühnen-Bund vgl. Keitz 2005.

49 Ackerknecht 1918, 5-18.

50 Kalbus 1922, 18. 
Unterstützung durch das im März 1915 am preussischen Kultusministerium gegründete 〈Zentralinstitut für Erziehung und Unterricht〉. Dieses Zentralinstitut wurde, wie viele andere gegen Ende des Krieges ohne parlamentarische Bewilligung gegründete Institutionen, durch die sogenannte <Jubiläumsstiftung> finanziert, die 1913 anlässlich des 25. Jahrestages der Krönung Wilhelms II. ins Leben gerufen worden war $^{51}$. In enger Kooperation mit dem Ministerium fand im April 1917 eine Schulung in den Räumen der Stettiner Urania statt, zu der alle mit Filmfragen befassten kommunalen Beamten deutscher Gemeinden und Städte mit über 10000 Einwohnern eingeladen wurden. Im Anschluss daran wurde im Juli 1917 der «Bilder-Bühnen-Bund Deutscher Städte> gegründet und kurz darauf ein ihm nachgeordneter «Lichtspielrat> geschaffen, der als Archiv für Lehr- und Dokumentarfilme dienen sollte. Dies gilt als erster Schritt zur Zentralisierung des Filmwesens, denn bald wurde der Lichtspielrat der Berliner Bildstelle des «Zentralinstituts für Erziehung und Unterricht> einverleibt. Ackerknechts ehemals kommunale Initiative war damit in einer Unterabteilung des preussischen Kultusministeriums aufgegangen. Von dem anfangs taktgebenden «Bilder-Bühnen-Bund Deutscher Städte> blieb «als Erinnerung» lediglich ein ehemaliges Vorstandsmitglied im Hauptausschuss der Berliner Bildstelle erhalten ${ }^{52}$.

Die Filmtheorie Ackerknechts war von der zeitgenössischen Literaturwissenschaft und Pädagogik inspiriert. Seine 〈Lichtspielreform〉 ist der Reformbühne der 1910er Jahre verpflichtet. Dort bemühten sich Schauspieler, ein einheitliches, ehrliches und wahres Bild ihrer Rollen im engen Sinne des Wortes zu 〈verkörpern〉. Ackerknechts im März 1918 erschienenes Buch «Das Lichtspiel im Dienste der Bildungspflege» enthielt die bis dahin umfangreichste Bibliographie zum Kino - Münsterbergs «The Photoplay» ist darin allerdings nicht gelistet. Ähnlich wie der Psychologe aus Harvard geht der Stettiner Bibliothekar in seiner Filmtheorie von der psychologischen Wirkung auf den Zuschauer aus: Bewegungsreize, so Ackerknecht, fesseln die Aufmerksamkeit des Betrachters.

Es leuchtet nun wohl ohne weiteres ein, dass das Besondere aller Wirkung des Lichtspiels, als des Trägers optischer Bewegungsreize, im Gefühlsmässigen liegt, genauer in der verhältnismässigen Stärke seiner gefühligen Faktoren, und dass jede bildungspolitische Verwertung des Lichtspiels vor allem diese ins Auge fassen sollte. ${ }^{53}$

Hier finden Ackerknecht und Münsterberg zu einer weitgehend identischen Schlussfolgerung: Inhalt muss über Emotion transportiert werden. Doch wo Münsterberg an die Suggestionskraft des Films glaubt, hofft Ackerknecht auf

51 Dietrich 1919, 9-11.

52 Kalbus 1922, 20.

53 Ackerknecht 1918, 38. 
Authentizität und die Beweiskraft des Bildes. Im Frühjahr 1918 liest sich eine seiner Einlassungen zur filmischen Dokumentation von Kriegsereignissen wie folgt:

Es war zweifellos ein höchst wertvoller Gedanke unserer Heeresleitung, ihre Kriegsaufnahmen nicht nur im Hinblick auf die militärwissenschaftliche oder auch auf die historische Verwertung zu machen und zu verwalten, sondern gleich die mächtigen moralischen Gefühlsimpulse, die von solchen Laufbildern ausgehen können, in den Dienst der Volkserziehung zu stellen. Ihre gefühlsmässige Wirkung wird übrigens in späteren Jahren bei einem Geschlecht, dass dem Krieg wieder unbefangener, weniger «abgebrüht» gegenübersteht, womöglich noch deutlicher zur Geltung kommen. ${ }^{54}$

In der zweiten Auflage desselben Aufsatzes, die zehn Jahre später erschien, fügte Ackerknecht hinzu:

Wie vorauszusehen war, haben die starken moralischen Antriebe, die von ihnen [den Kriegsaufnahmen, P. O.] ausgehen, jetzt, nachdem der Kampf des «Durchhaltens» längst vorüber ist, bei vielen, ja vielleicht bei den meisten Beschauern keine kriegsbejahende Wirkung mehr, sondern eine kriegsverneinende. ${ }^{55}$

Die kritische Haltung, die bereits aus den Formulierungen der ersten Auflage zu ahnen ist, mag ein Grund dafür gewesen sein, dass der Bilder-Bühnen-Bund «in den Hintergrund gedrängt wurde», wie der Wissenschaftliche Referent der Ufa-Kulturfilmabteilung, Oskar Kalbus (1890-1987), die Eingliederung der von Ackerknecht errichteten Strukturen in die preussische Bildstelle 1922 euphemistisch protokollierte ${ }^{56}$.

\section{«Erster Vorschlag: Die Reichszentrale für Heimatdienst behält entscheidenden Einfluss auf den Propagandafilm.» Politische Einfluss- nahme auf Lehr- und Kulturfilme ab 1918}

Nach dem Ende des Krieges herrschte eine für Ämter und Filmindustrie chaotische Lage: Die Ufa war weiterhin im Besitz des Staates. Dabei bestand allgemeiner Konsens darüber, dass die Monopolisierung auf Betreiben Ludendorffs unrechtmässig vonstatten gegangen war und rückgängig zu machen sei. Nach der Revolution befassten sich fünf unabhängige Stellen der Weimarer Regierung mit der Produktion von Filmen ${ }^{57}$ :

54 Ackerknecht 1918, 45.

55 Ackerknecht 1928, 22. In diesem Band wurde der Artikel des Jahres 1918 angeblich «unverändert» wieder abgedruckt, die oben wiedergegebenen Zitate zeigen, wie Ackerknecht seine Andeutung von 1918 für die Version von 1928 konkretisierte.

56 Kalbus 1922, 20.

57 Die folgende Aufstellung und die zugehörigen Zitate stammen aus dem Druck: Zentrale für den Heimatdienst: Regierung und Film. Berlin, den 12. September 1919. Akten betreffend Film-Angelegenheiten, Alte Reichskanzlei. Bundesarchiv Berlin, Bestand R 43 I/ 2497, Blatt $142-145$. 
1. Das Filmdezernat der Presseabteilung des Demobilisierungsamtes. Die Spielfilme «Mutter Erde», «Tod der Freiheit», und «Ein kleiner Siedlungsfilm» sind die einzige Hinterlassenschaft dieser Abteilung.

2. Das Filmdezernat des Auswärtigen Amtes.

3. Die Reichsfilmstelle, in der die ehemaligen Beamten der Bufa den Verleih der Vorkriegsfilme verwalteten. Produziert wurden dort nur noch Lichtbildserien für Diavorträge.

4. Das Filmdezernat der Reichskanzlei ${ }^{58}$.

5. Das Filmdezernat der Reichszentrale für Heimatdienst.

Gegründet acht Monate vor Ende des Krieges, hatte dieses Dezernat nach eigenen Angaben «Film und Lichtbild als Mittel zur politischen Beeinflussung zu bearbeiten gehabt». Nach dem Krieg entstanden in seinem Auftrag eine Reihe von Filmen und Lichtbildserien, in denen die Parlamentarische Demokratie und das Frauenwahlrecht erläutert wurden. Mit diesen Produktionen präsentierte sich die Reichszentrale für den Heimatdienst als anpassungsfähig. Noch die heutige Bonner Bundeszentrale für Politische Bildung sieht ihre historischen Wurzeln in dieser Institution. Als Atavismus aus Kriegszeiten, der schnellstmöglich abzuwickeln sei, wurden die in staatlichem Besitz befindlichen Filmproduktionsgesellschaften von der Regierung aber nicht betrachtet. Im Gegenteil: Am 2. Februar 1919 schloss das ReichsFinanzministerium einen Vertrag mit der Ufa, in dem die Regierung ihre Ansprüche geltend machte, aufgrund des von ihr gehaltenen Aktienpaketes nicht nur ökonomisch, sondern auch inhaltlich Einfluss auf die Kulturfilme zu nehmen; in §7 des Abkommens heisst es:

Die Ufa verpflichtet sich andererseits, ihr vom Reich zugehenden Anregungen und Wünschen bezüglich der Herstellung oder der Verbreitung gewisser Filme oder Lichtbilder in weitgehendem Masse entgegenzukommen. ${ }^{59}$

Erst nach Unterzeichnung dieser Erklärung verfügte der Reichsschatzminister die Übergabe der Bestände der Reichsfilmstelle an die UniversumFilm-Aktiengesellschaft ${ }^{60}$. Ganz ohne Widerstand vollzog sich diese Proforma-Privatisierung nicht. Das Quasi-Monopol der Ufa wurde von dem Zentralverband der Film- und Kinoangehörigen Deutschlands mit Argwohn

58 Dieser Stelle war zunächst die zentrale Rolle zugedacht worden, sämtliche Propagandaaktivitäten der fragilen Demokratie zu koordinieren. Vor allem gegen ihre Einflussnahme richtete sich die Lobbyarbeit der Filmbranche. Produziert hatte das Filmdezernat der Reichskanzlei bis Herbst 1919 zwei kurze Filme, der eine trug den Titel «Gegen den Gewaltfrieden», der andere hiess «Die Sozialisierung marschiert».

59 Kalbus 1922, 22.

60 Zentrale für den Heimatdienst: Regierung und Film. Druck, Berlin, den 12. September 1919. Akten betreffend Film-Angelegenheiten, Alte Reichskanzlei. Bundesarchiv Berlin, Bestand R 43 I/ 2497, Blatt 142. 
betrachtet. Dieser Verband vertrat insbesondere die Interessen von Kinobetreibern und zunehmend auch die der verbliebenen bzw. neugegründeten (Klein-)Unternehmen der Filmindustrie. In Revolutionszeiten hatte man sich den schlagkräftigen Namen «Deutsche Filmgewerkschaft zugelegt und unter dieser Bezeichnung den Demobilisierungskommissar dazu aufgefordert, eine avisierte Fusion der staatlich alimentierten Ufa mit der $\triangleleft$ Deutschen Bioskop Babelsberg $>$ zu verhindern ${ }^{61}$. Nun sandte dieselbe Organisation ein Schreiben an die Reichskanzlei, in dem sie zu erkennen gab, dass ihr der geheime Gründungsprozess der Ufa nicht verborgen geblieben war:

Es muss schließlich noch darauf hingewiesen werden, dass das Reich an der Ufa mit einem Kapital von 7,5 Millionen Mark beteiligt ist. Diese, in noch von der vorrevolutionären Regierung, der Öffentlichkeit auch heute noch unbekannten Geheimverträgen, eingegangene Verbindung legt öffentliche Gelder in einem durchaus privatkapitalistischen Interessen dienenden Unternehmen fest. ${ }^{62}$

In der Reichskanzlei wurden Vorschläge gesammelt, wie auf das Schreiben zu reagieren sei, zur Absicherung wurden Reichsfilmstelle und Schatzminister hinzugezogen ${ }^{63}$. Die Herren entschieden, besser ausweichend zu antworten:

Herr Unterstaatssekretär Walther empfahl, auch ferner eine eingehende Äußerung auf die einzelnen Punkte der Eingabe des Zentralverbands der Filmangehörigen zu unterlassen und nur generell darauf zu antworten [...]. Über die Beteiligung des Reiches an der Ufa schweben zur Zeit Erwägungen. Es würde sich aber nicht empfehlen, in dem Antwortschreiben an den Zentralverband der Kinoangehörigen hierüber etwas verlauten zu lassen. ${ }^{64}$

Im stillen aber begann die unter Druck geratene Regierung zu handeln, zumal sich kritische Berichte in der Presse mehrten. Die Lichtbildbühne schrieb:

Die Regierung hat es an gutem Willen nicht fehlen lassen. Sie hat das Bild- und Filmamt demobilisiert und es als Reichsfilmstelle zu neuem Leben erweckt. Kein Mensch ist in der Lage, über die Befugnisse der Stelle klare Auskunft zu geben. [...] Sie hat bei der Reichskanzlei ein Filmdezernat errichtet, über dessen Funktionen und Kompetenzen allgemeine Unklarheit herrscht. Ist es nur eine politische Informationsstelle für die Reichsregierung

61 Schreiben des Zentralverbands der Film- und Kinoangehörigen Deutschlands an den Unterstaatssekretär der Reichskanzlei vom 23. Oktober 1919, Akten betreffend Film-Angelegenheiten, Alte Reichskanzlei. Bundesarchiv Berlin, Bestand R 43 I/ 2497, Blatt 150-152. Der von der Filmgewerkschaft angegebene Wert von 7,5 Millionen Mark hatte sich mittlerweile beträchtlich erhöht, allein die kleinere Produktionsgesellschaft «Deutsche Bioskop Babelsberg> wurde Mitte 1919 von der Reichskanzlei auf einen Wert von 14 Millionen Mark taxiert.

62 Ebendort (wie Fussnote 61).

63 Der Unterstaatssekretär in der Reichskanzlei an den Reichsschatzminister. Handschriftliche Notiz vom 6. Dezember 1919. Akten betreffend Film-Angelegenheiten, Alte Reichskanzlei. Berlin, Bestand R 43 I/ 2497, Blatt 165.

64 Reichsfilmstelle an den Herrn Unterstaatssekretär in der Reichskanzlei. Protokoll vom 11. November 1919. Akten betreffend Film-Angelegenheiten, Alte Reichskanzlei. Bundesarchiv Berlin, Bestand R 43 I/ 2497, Blatt 163-164. 
oder stellt es eine amtliche Verbindung zur Branche her, die auch in wirtschaftliche oder geistige Fragen der Filmwirtschaft eingreift? Darauf ist eine Antwort nicht zu erhalten ${ }^{65}$

Bereits im September 1919 setzten Beratungen über die Neuordnung der deutschen Filmwirtschaft ein: Zu diesem Zweck hatte die Reichszentrale für den Heimatdienst eine Vorlage verfasst, die heute im Berliner Bundesarchiv im Aktenbestand der alten Reichskanzlei liegt. Unter dem Titel «Regierung und Film» wurden hier Entscheidungen mit weitreichenden Folgen für den Kulturfilm vorbereitet. Das Papier prophezeite der Filmindustrie Gewinne wie kaum einem anderen Wirtschaftszweig. Selbstkritisch merkte die Reichskanzlei an, dass in der amtlichen Verwaltung der deutschen Filmindustrie Misswirtschaft herrsche, und warnte vor weiteren Kampagnen des Zentralverbands der Kinoangehörigen. «Die Regierung trennt sich von der Ufa», lautete daher ein mit Dringlichkeit vorgebrachter Vorschlag ${ }^{66}$, der sukzessive in den folgenden anderthalb Jahren verwirklicht werden sollte.

Auf der Liste der Empfehlungen war das aber nur Vorschlag Nummer drei. Der erste Vorschlag ist weit weniger bekannt, er lautet: «Die Reichszentrale für Heimatdienst (R.f.H.) behält entscheidenden Einfluss auf den Propagandafilm $»^{67}$. «Handzettel, Flugblatt, Broschüre und Plakat», so lautete die Begründung, «sind als Aufklärungsmittel durch übermäßige Beanspruchung entwertet worden. Dementsprechend sind Lichtbild und Film die entscheidenden Propagandamittel der Zukunft, zumal die Massen dem Film geradezu verfallen sind und deshalb durch ihn am besten ergriffen werden können.» ${ }^{68}$ Die Produktion von Kulturfilmen solle daher von einem mit weitreichenden Befugnissen ausgestatteten Reichsfilmamt bedarfsgerecht geplant und überwacht werden. Zur Einrichtung einer solchen Behörde kam es nie, obwohl sie kontinuierlich über das gesamte Bestehen der Weimarer Republik hinweg gefordert wurde ${ }^{69}$. Das Ziel, die staatliche Kontrolle der Kulturfilme, wurde dennoch erreicht. Das geschah mit einem weit akkurateren Instrumentarium als dem der kommunalen polizeilichen Zensur, auf die die Regierung keinen unmittelbaren Einfluss hatte, die aber nach dem

65 Lichtbildbühne Nr. 33 vom 16.8.1919. Zitiert nach: Zentrale für den Heimatdienst: Regierung und Film. Druck, Berlin, den 12. September 1919. Akten betreffend Film-Angelegenheiten, Alte Reichskanzlei. Bundesarchiv Berlin, Bestand R 43 I/ 2497, Blatt 143.

66 Zentrale für den Heimatdienst: Regierung und Film. Druck, Berlin, den 12. September 1919. Akten betreffend Film-Angelegenheiten, Alte Reichskanzlei. Bundesarchiv Berlin, Bestand R 43 I/ 2497, Blatt 142.

67 Ebendort (wie Fussnote 66).

68 Zentrale für den Heimatdienst: Regierung und Film. Druck, Berlin, den 12. September 1919. Akten betreffend Film-Angelegenheiten, Alte Reichskanzlei. Bundesarchiv Berlin, Bestand R 43 I/ 2497, Blatt 145.

69 Vergleiche Kalbus 1922, 46, sowie: Kaufmann 1931, 42. 
Reichslichtspielgesetz vom Mai 1920 von den Film-Oberprüfstellen in Berlin und München zentral überwacht wurde.

Ein differenziert einsetzbares Mittel, um die Vorführung erwünschter Kulturfilme zu befördern, war die Regulierung der Lustbarkeitssteuer, die Kinobetreiber an die Kommunen zu entrichten hatten. Jede Gemeinde besass das Recht, die Höhe dieser Abgabe selbst zu bestimmen. Die Reichsregierung drängte, Lichtspielhäuser, die in ihr Programm Kulturfilme aufnahmen, von der Steuer zu befreien. 1923 wurde vom Reichsrat eine Norm erlassen, die «Steuerstellen verpflichtet, [...] für Veranstaltungen, bei denen der künstlerische oder volksbildende Charakter überwiegt, Steuerermässigungen zu gewähren $»^{70}$. Mit diesem Rahmengesetz gelang es, die staatliche Kulturfilmförderung im Sinne der Reichsregierung auf kommunaler Ebene flächendeckend umzusetzen.

Welche Lehr- und Kulturfilme anerkannt wurden, entschied in Preussen eine Beratungs- und Prüfstelle, die unmittelbar dem Kultusministerium unterstand. Begeistert schwärmte der Leiter der Ufa-Kulturfilmabteilung, der Arzt Nicholas Kaufmann (1892-1970), wie selbständig diese Bildstelle agiere, die nach ihren Leitern zunächst «Lampe-> und später <Günther-Ausschuss〉 genannt wurde: Ohne jeden bürokratischen Zwang fördere sie im Interesse des Staates die Hebung und Weiterentwicklung des Volkes, indem sie beratend, regulierend und anregend in die Herstellung der Filme eingreife. «Ihrem Statut nach hat sie nicht nur die von der Industrie fertiggestellten Filme zu begutachten, sondern kann auch von der Industrie bei der Auswahl der Themen und bei der Festsetzung der Art ihrer Durchführung zu Rate gezogen werden.» Jeder Freund des Kulturfilms müsse die Forderungen mit Besorgnis verfolgen, die «einzigartig glückliche Organisation der Bildstelle grundlegend zu ändern» ${ }^{11}$. In der Tat hatte sich Protest erhoben. Bayern war aus der Praxis ausgestiegen und vergab eigene Zertifikate. Besonders bissig nahm Rudolf Arnheim (1904-2007) die Praxis der Bildstelle in der Berliner Weltwoche aufs Korn. Filme, die im Lampe-Ausschuss durchfielen, hatten ohne Kulturfilmschein keine Chance im Verleih. Ein experimenteller Städtefilm des Science-Fiction-Autors Heinrich Hauser (19011955) über Chicago war abgelehnt worden, und Arnheim ironisierte die biedere Empörung der Pädagogen, die über sein Schicksal entschieden hatten: «Den Herren zittert die Lippe. Der Film wird nicht nur abgelehnt, nein, er ist eine Zumutung, ein Tiefschlag in die edelsten Weichteile der Pädagogik.» ${ }^{72}$ Kurt Tucholsky (1890-1935) amüsierte sich derweil über die Text-

70 Seeger 1924, 72-75.

71 Kaufmann 1931, 40.

72 Arnheim 1932. 
lastigkeit ambitionierter Kulturfilme. Seine Kritik an den zahlreichen erklärenden Sätzen auf eingeblendeten Zwischentafeln trug den Titel «Der Lesefilm» ${ }^{73}$.

Prüfstelle und Steuerbefreiung bildeten in ihrer Kombination eine erhebliche Einflussmöglichkeit des Staates auf die Kulturfilme. Es bleibt festzuhalten, dass das preussische Kultusministerium, und hier die von dem Mediziner Eduard Dietrich geleitete Abteilung, für jene Verordnung verantwortlich zeichnete ${ }^{74}$, die am 15. Januar 1919 die Einrichtung der Zentralen Beratungs- und Prüfstelle für Lehrfilme anordnete. Zur historischen Orientierung hilft es, sich die damaligen Tagesereignisse zu vergegenwärtigen. An diesem Tag fanden die Ausläufer der Novemberrevolution mit der Ermordung Rosa Luxemburgs (1871-1919) und Karl Liebknechts (18711919) einen blutigen Höhepunkt, und am folgenden Sonntag sollten die ersten Reichstagswahlen abgehalten werden. Lehrfilm, Volksbelehrungsfilm und Propagandafilm wurden mit dem Dekret der Medizinalabteilung an eine zentrale Kontrollstelle verwiesen, die am 2. Mai 1919 zu ihrer konstituierenden Sitzung zusammentrat. Medizinalrat Dietrich sorgte dafür, dass die Kontrollstelle nicht zu passiver Bewertung bereits abgedrehter Filme verdammt war: Mitglieder des Ausschusses erhielten die Freiheit, auf persönliche Initiative mit Produktionsgesellschaften in Verhandlungen zu treten, um von ihnen erwünschte Lehrfilme in Auftrag zu geben ${ }^{75}$. Der Leiter des Zentralinstitutes, Ludwig Pallat (1867-1946), machte deutlich, dass von der Industrie erwartet werde, sich «in Fragen des belehrenden Jugendunterhaltungsfilms um Rat für pädagogische Zurechtrückung an die Bildstelle» zu wenden ${ }^{76}$.

Waren es die Sachverständigen, die hier Politik gestalteten, oder kontrollierten die leitenden Regierungsräte die in ihrem Gremium vertretenen Mediziner und Pädagogen? In späteren Jahren entstand ein weiteres, übergeordnetes Instrument, das das Wechselspiel von Politik und sachverständigen Pädagogen und Medizinern weiterbeförderte: der $<$ Reichsausschuss für Hygienische Volksbelehrung>. 1926 konstituierte er sich als Unterabteilung der seit 1922 bestehenden «Arbeitsgemeinschaft sozialhygienischer Reichs-

73 Panter 1927.

74 Dabei überging das Ministerium die bereits am Kaiserin-Friedrich-Haus für ärztliche Fortbildung bestehende 〈Zentralstelle für medizinische Filme〉. Es fiel dem Vertreter der Heeresleitung, Oberstabsarzt Maeker, in der konstituierenden Sitzung des Hauptausschusses der Beratungs- und Prüfstelle für Lehrfilme durchaus auf, dass der Leiter des ärztlichen Fortbildungswesens, Curt Adam (1875-1941), nicht vertreten war. Redebeitrag Maeker. Bericht über die konstituierende Sitzung des Hauptausschusses der Beratungs- und Prüfstelle für Lehrfilme. Protokoll vom 2. Mai 1919. Akten betreffend Film-Angelegenheiten, Alte Reichskanzlei. Bundesarchiv Berlin, Bestand R 43 I/ 2497, Blatt 17-22.

75 Redebeitrag Dietrich, ebendort (wie Fussnote 74).

76 Redebeitrag Pallat, ebendort (wie Fussnote 74). 
fachverbände>. Er erarbeitete didaktische Konzepte für die Gesundheitsaufklärung in Presse, Film und Radio. In seiner Zusammensetzung ähnelte auch dieses Gremium, ebenso wie der medizinische Ausschuss der «Bildstelle`, frappierend dem Kuratorium der Dresdner Hygiene-Ausstellung von 1911. Und auch der Name ihres Initiators stimmt mit dem Koordinator von preussischen Interessen und medizinischem Sachverstand auf der Dresdner Ausstellung überein - Eduard Dietrich. Das Zusammenspiel einzelner Vereinigungen, viele von ihnen bereits zur Kaiserzeit gegründet, mit Vertretern des preussischen Volkswohlfahrtsministeriums und des Reichsinnenministeriums ermöglichte es der Arbeitsgemeinschaft sozialhygienischer Reichsfachverbände, meinungsbildend auf die Politik einzuwirken und die Eckdaten der hygienischen Volksbelehrung festzulegen.

Gremien und Ausschüsse, die 1911 erste Propagandakonzepte für Ausstellungstafeln, Lehrdias und Lichtspiele zur hygienischen Volksbelehrung auf der Dresdner Hygiene-Ausstellung und für die frühen Kriegsausstellungen erarbeitet hatten, legten nun die Grundlagen für die Sonderstellung des «deutschen Kulturfilms» in der Weimarer Republik. Etwas getragen brachte Oskar Kalbus 1924 die Festigung dieser Strukturen während des Ersten Weltkriegs in seiner kurzen Geschichte der deutschen Lehrfilmbewegung auf den Punkt:

So verhängnisvoll der große Weltkrieg auch in seinen Folgen für die wirtschaftliche und politische Lage in Deutschland war, für den deutschen Lehrfilm war er Reformer und Schöpfer zugleich. ${ }^{77}$

\section{«Krüppelnot und Krüppelhilfe»: der erste «abendfüllende Kulturfilm» der Ufa als Beispiel für die Entstehung eines neuen Filmgenres}

Der Film «Krüppelnot und Krüppelhilfe» spiegelt die frühen Kulturfilme der Weimarer Republik in vielen Facetten ${ }^{78}$. Uraufgeführt am 2. September 1920 in den Kammerlichtspielen am Potsdamer Platz, ist er ein frühes Beispiel für den fortschreitenden Professionalisierungsprozess des deutschen Kulturfilms. Der Film sollte Einfluss auf eine politische Debatte nehmen, die bis in die Zeit vor Ausbruch des Krieges zurückreicht. Auf sie muss zur Erläuterung des unmittelbaren Kontextes des Films «Krüppelnot und Krüppelhilfe» kurz eingegangen werden. Im Frühjahr 1914 hatte der bereits mehrfach erwähnte Leiter der preussischen Medizinalabteilung Eduard

77 Kalbus 1924a,1-13.

78 «Krüppelnot und Krüppelhilfe». Deutschland 1920. Produktion Ufa Kulturabteilung. 35 mm, s/w, 1.062 m, Regie Nicholas Kaufmann. Kopie im Bundesarchiv-Filmarchiv. 
Dietrich einen Gesetzesentwurf konzipiert, der eine Meldepflicht und zwangsweise Heimunterbringung für körperbehinderte Kinder vorsah und Städte und Kommunen dazu verpflichtete, bei Mittellosigkeit der Eltern für die Behandlung und Ausbildung der Kinder nach dem Armenrecht zu sorgen. Es war ein Versuch, sozialdemokratischen Forderungen entgegenzutreten, die dafür warben, die medizinische Versorgung Körperbehinderter durch den preussischen Staat zu alimentieren ${ }^{79}$. Unter den Schutz der allgemeinen Krankenversicherungen fielen körperliche Behinderungen nicht, da sie als sogenannte «Statische Leiden» und nicht als Krankheiten angesehen wurden ${ }^{80}$. Die preussische Regierung wünschte sich eine Berücksichtigung der Körperbehinderten im Armenrecht, da bei dieser Konstruktion die anfallenden Sozialkosten zu Lasten der Kommunen gingen. 1914 verhinderte der Beginn des Ersten Weltkrieges eine Parlamentsdebatte über den entsprechenden Gesetzentwurf des Ministerialrates Dietrich.

1919 wurde der Düsseldorfer Arzt und preussische Abgeordnete Arthur Schlossmann (1867-1932) vorgeschickt, den Entwurf erneut einzubringen, diesmal über den Ausschuss für Bevölkerungspolitik der preussischen Landesversammlung. Gleich zu Beginn des Verfahrens ergaben sich Probleme mit den neuen demokratischen Gepflogenheiten; Selbsthilfeverbände verschafften sich Gehör und protestierten gegen die Meldepflicht und insbesondere gegen die zwangsweise Heimunterbringung körperbehinderter Kinder und Jugendlicher ${ }^{81}$. Als Bestimmung des Armenrechts hätte das Krüppelfürsorgegesetz einen solchen Passus nicht erhalten dürfen. Ähnlich drastische Bestimmungen wurden sonst lediglich für seuchenpolizeiliche Massnahmen erwogen. Darüber hinaus durfte eine Norm, die in die Finanzen der Kommunen eingriff, nicht über den Bevölkerungsausschuss eingebracht werden, sie hätte den Gemeindeausschuss passieren müssen. In erregter Debatte wurde das Gesetz dennoch am 6. Mai 1920 in der ursprünglich vorgesehenen Form verabschiedet, da die Parlamentarier über alle Parteigrenzen hinweg dringenden Handlungsbedarf sahen ${ }^{82}$. Trotz der Proteste von Gemeinden und Selbsthilfeverbänden trat es am 1. Oktober 1920 in Kraft. Der Gesetzgebungsprozess war a priori juristisch anfechtbar

79 «Ich meine, was da möglich ist und was den Gemeinden möglich ist, das sollte auch dem Preussischen Staat möglich sein.» Redebeitrag des sozialdemokratischen Abgeordneten Hirsch aus Berlin in der 62. Sitzung des preussischen Abgeordnetenhauses am 30.4.1910. Geheimes Staatsarchiv Preussischer Kulturbesitz. Bestand. Rep. VIII B Ministerium für Wissenschaft, Kunst und Volksbildung, Nr. 1675, B1. 224.

80 Köhne 1892, 83.

81 Schlossmann 1920, 29.

82 Das Gesetz, betreffend die öffentliche Krüppelfürsorge vom 6. Mai 1920 ist wiedergegeben bei Wendenburg 1929, 129-131. 
und auch die gesetzliche Meldepflicht hätte, wäre dagegen geklagt worden, nicht aufrechterhalten werden können. Eine propagandistische Begleitung des Gesetzes durch filmische Mittel bot sich daher an. Parlamentarier wurden sowohl an den Originalschauplatz des Films, in das Oskar-HeleneHeim, eingeladen als auch zu einer Sondervorführung von «Krüppelnot und Krüppelhilfe». Wie sehr der Film überzeugt haben mag, spiegelt sich in dem Medienecho auf die Premierenvorführung. Der Kritiker des Filmkuriers vom 3. September 1920 fand besonders viele Superlative:

Als Kunstwerk ist er Kunstwerk über jedem Kunstwerk; ungewollt; Drama über jedem Drama, Lyrik über jeder Lyrik, Vermittler einer reinsten verklärtesten Erschütterung. ${ }^{83}$

Erwünschter noch als die positiven Besprechungen durch Fachzeitschriften war die Tatsche, dass «Krüppelnot und Krüppelhilfe» Eingang in die politische Tagesberichterstattung fand ${ }^{84}$. Die Uraufführung fiel zeitlich mit einem Kongress der Deutschen Gesellschaft für Krüppelfürsorge zusammen. Vorsitzender dieses Zusammenschlusses von Kinderheimen, orthopädischen Kliniken, kirchlich getragenen Wohnheimen, Armenfürsorgestellen und Behörden war der preussische Ministerialdirektor Eduard Dietrich, Schriftführer der Direktor des Berliner «Oskar-Helene-Heims für die Heilung und Erziehung gebrechlicher Kinder», Konrad Biesalski (1868-1930), in dessen Anstalt der Film gedreht worden war. Schon auf der Dresdner Hygiene-Ausstellung liess Biesalski Filme vorführen, die er ab 1910 in Eigenregie produziert hatte. Grosse Teile dieser Filme finden sich unverändert in dem Ufa-Kulturfilm «Krüppelnot und Krüppelhilfe» wieder. Diese Szenen sind ausschliesslich dokumentarisch: Operationen, gymnastische Übungen und Kinder, die an Werkbänken, Webstühlen oder Brennöfen stehen und damit den Erfolg ihrer Berufsausbildung in Biesalskis Anstalt demonstrieren. Hinzu kamen weitere Szenen, die in unregelmässigen Abständen in den Jahren 1911 bis 1919 aufgenommen worden waren. Zunächst arbeitete Biesalski mit diversen Filmfirmen zusammen, 1914 stiftete ihm ein Berliner Industrieller eine eigene kinematographische Anlage. Die damit hergestellten Aufnahmen sind in der heute erhaltenen Version von «Krüppelnot und Krüppelhilfe» enthalten. Die im Berliner Bundesfilmarchiv aufbewahrte Fassung entspricht ihrer Länge nach genau der Version, die im September 1920 uraufgeführt wurde.

83 A-j., «Lehrfilme zum Krüppelkongress. Probevorführungen der Ufa», Filmkurier 196 vom 3. September 1920.

84 «Krüppelheime und Krüppelschulen. Der zweite Tag des Kongresses», Berliner Morgenpost Nr. 229/1920 vom 3. September 1920; «6. Deutscher Kongress für Krüppelfürsorge», Berliner Volkszeitung 416/1920 vom 3. September 1920; «Deutscher Kongress für Krüppelfürsorge», Deutsche Allgemeine Zeitung 432/1920 vom 4. September 1920. Ich danke Jeanpaul Goergen, Cinegraph Babelsberg, für die Überlassung dieser Zeitungsseiten. 
In «Krüppelnot und Krüppelhilfe» mischen sich klassische Lehrfilm-Elemente mit fröhlichen Szenen aus dem Anstaltsleben. Der Film zeigt neurologische Symptome bei Gangbildern, didaktische Unterweisungen für die Ausbildung von Krankengymnastinnen und dann wieder Sequenzen mit Patienten, die ohne direkten Bezug zu medizinischen Interventionen stehen. Vor allem die Alltagsszenen sollen den Zuschauer «gefangen nehmen», wie einer zeitgenössischen Beschreibung des Films zu entnehmen is ${ }^{85}$.

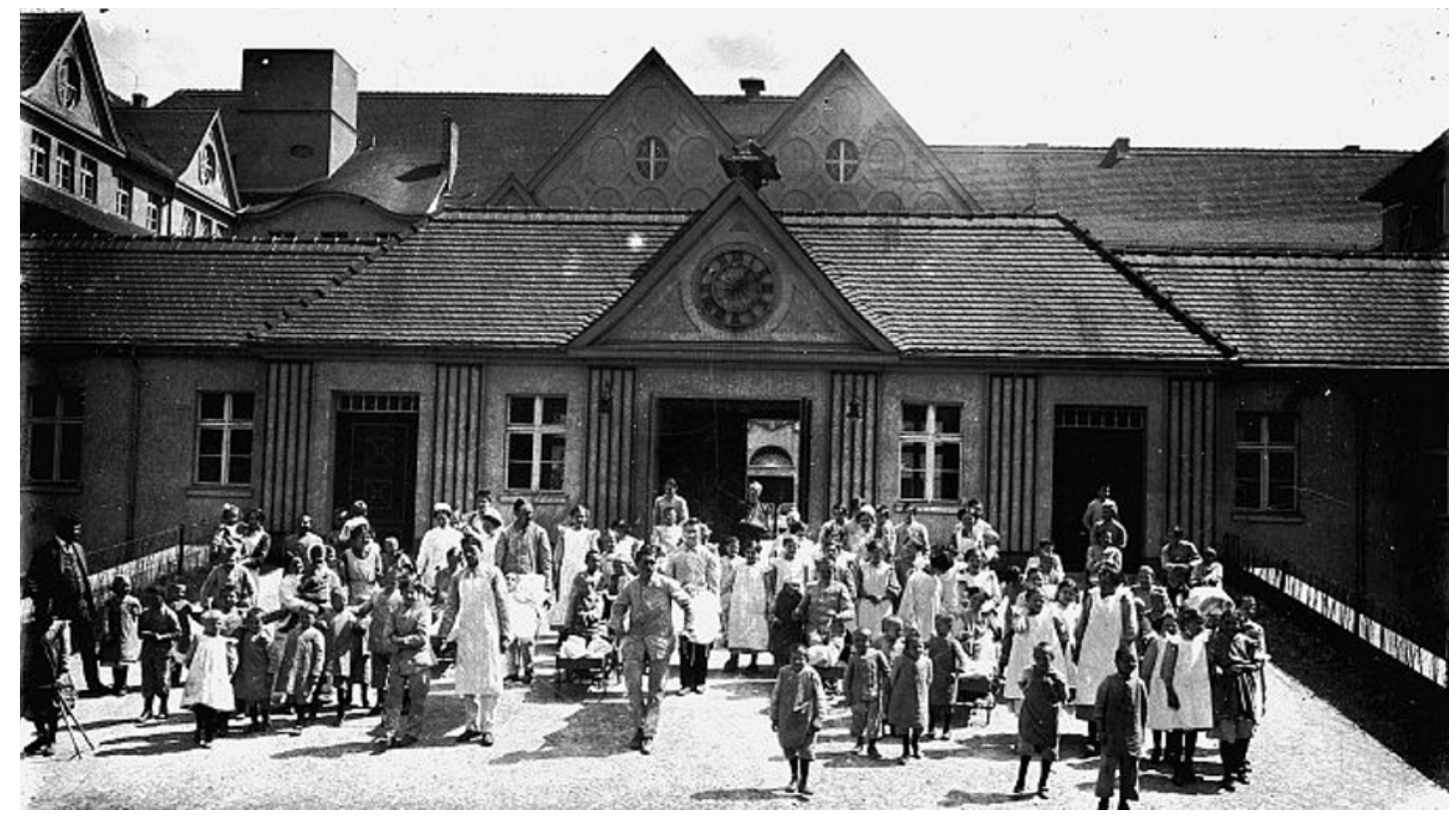

Abb. 2. Szene aus dem Film «Krüppelnot und Krüppelhilfe», Glasnegativ aus dem Jahr 1914.

Die eindrucksvollste Sequenz des Films von 1920 zeigt den Auszug körperbehinderter Kinder aus dem Hauptgebäude in den Wald hinter der Anstalt. Einen dunklen Gang entlang kommen sie auf die Kamera zu. Einige gehen an Krücken, andere werden von ihren Mitpatienten in Rollstühlen gefahren. Pflegerinnen schieben die Säuglinge auf fahrbaren Tragen am Kameramann vorbei. Ihnen folgen Schwestern, Ärzte und Lehrer. In der nächsten Einstellung hat sich der lange Zug von etwa 80 Personen auf einer breiten Rampe verteilt, die vom ersten Stock des Gebäudes in den Garten führt (Abb. 2). Von hier lösen sich einige Kinder aus dem Pulk. Sie laufen, immer schneller werdend, hinunter in den Wald. Die Treppenszene ohne Stufen wurde nicht, wie bisher angenommen, 1920 unter der Leitung des UfaMitarbeiters Nicholas Kaufmann aufgenommen, der bis heute als Regisseur von «Krüppelnot und Krüppelhilfe» gilt, sondern bereits 1914 von dem Ärzt- 
lichen Direktor der Anstalt, Konrad Biesalski ${ }^{86}$. Biesalski war der Chirurg, der 1896 zu Beginn seiner Karriere im genannten Optikergeschäft Messter an der Berliner Friedrichstrasse eine diagnostische Röntgenfotografie hatte anfertigen lassen. Seit der Dresdner Hygiene-Ausstellung von 1911 sass er als Berater in den vom preussischen Kultusministerium kontrollierten Ausschüssen zu Propagandafragen. Er hatte die Darstellung der sogenannten Kriegskrüppelfürsorge in den Kriegsausstellungen des Ersten Weltkriegs verantwortet, er war Mitglied im medizinischen Ausschuss der Bildstelle ${ }^{87}$, im preussischen Landesgesundheitsrat ${ }^{88}$ und im Reichsausschuss für hygienische Volksbelehrung ${ }^{89}$. Mit Medizinalrat Dietrich verband Biesalski eine enge Freundschaft, beide hatten in Halle studiert und waren Mitglieder derselben korpsstudentischen Verbindung. Das umstrittene Gesetz zur Krüppelfürsorge trug Biesalskis Handschrift, einige der darin enthaltenen Formulierungen stammen wortwörtlich von ihm. Nach dem Inkrafttreten der Norm bestimmte seine Klinik als «Zentral-Fortbildungs- und Forschungsanstalt für die Krüppelfürsorge in Preussen und im Deutschen Reiche> massgeblich den politischen Kurs der Gesundheits- und Sozialfürsorge für Körperbehinderte während der Zeit der Weimarer Republik ${ }^{90}$.

Das Entsetzen war gross, als die Zensurbehörde im Dezember 1920 die öffentliche Vorführung des Films vor Kindern und Jugendlichen untersagte ${ }^{91}$. Noch Jahre später ist dem wissenschaftlichen Referenten der Ufa sein Ärger über die in seinen Augen bornierte Berliner Polizeizensur anzumerken, die in der Präsentation nackter Leiber und «verkrüppelter Gliedmassen» eine sittliche Gefährdung erkannte: «Es hätte überhaupt gesetzlich geregelt werden müssen, dass Lehrfilme nur durch die Bildstelle am Zentralinstitut zu zensieren sind», schrieb Oskar Kalbus im Jahr 1922 $2^{92}$. Dieser Vorschlag war tatsächlich 1919 in der Reichskanzlei formuliert worden ${ }^{93}$, doch es gelang nicht, der Bildstelle auch noch polizeiliche Befugnisse zuzuweisen. So entstand eine paradoxe Situation. In der Öffentlichkeit wurde die Filmzensur

86 Zur Datierung und Zuschreibung der einzelnen Szenen von «Krüppelnot und Krüppelhilfe»: Osten 2008.

87 Dietrich 1919, 9-11.

88 Konrad Biesalski an den Ministerialdirektor Dr. Krone. Schreiben vom 11. August 1926. Geheimes Staatsarchiv Preussischer Kulturbesitz, Berlin Rep. 76 VIII B, Nr. 1677, Bl. 291-292.

89 Reichsausschuss für hygienische Volksbelehrung 1930.

90 Vgl. Osten 2004, 212-227.

91 Entscheidung der Filmprüfstelle Berlin über den Film «Krüppelnot und Krüppelhilfe» vom 6.12.1920. Archiv des Deutschen Filminstituts Frankfurt a. M., Bestand: Zensur, Filmprüfstelle Berlin B.00889. Online einsehbar über: http://www.deutsches-filminstitut.de/filme/ f035280.htm.

92 Kalbus 1922, 25.

93 Akten betreffend Film-Angelegenheiten. Vom 27. Mai 1919 bis zum 20. Oktober 1920. Bundesarchiv Berlin, Bestand R43 I, 2497. 
als das Organ staatlicher Einflussnahme schlechthin angesehen. In Wahrheit entzog sich die Arbeit dieser Behörde fast vollständig der staatlichen Direktive. Die Zensur war eines der wenigen unabhängigen Elemente im komplexen Zulassungsverfahren für Kulturfilme.

Erst im Januar 1921 erreichte Nicholas Kaufmann durch persönliche Vorsprache bei der neugeschaffenen Film-Oberprüfstelle die vollständige Freigabe seines Werks ${ }^{94}$. Es kann davon ausgegangen werden, dass «Krüppelnot und Krüppelhilfe» in jeder seiner Evolutionsstufen - von den frühesten Aufnahmen aus dem Jahr 1910 bis zu seiner Uraufführung am Potsdamer Platz - den jeweiligen Stand der zeitgenössischen filmischen Mittel im Medizinbereich repräsentierte. Das gilt für jede Sequenz für sich genommen. Aufnahmen aus dem Babysaal zeigten «allerlei niedliche Szenen» ${ }^{95}$, sie dienten der Werbung privater Spenden. Vorher-nachher-Bilder von operierten Patienten wurden auf Kongressen vorgeführt, und zahlreiche, in den Werkstätten und Lehrlingsbetrieben der Anstalt aufgenommene Szenen sollten die kommunale Armenfürsorge überzeugen, in die Ausbildung und Schulung körperbehinderter Jugendlicher zu investieren. All diese Elemente von «Krüppelnot und Krüppelhilfe» wurden über einen Zeitraum von zehn Jahren hinweg jeweils für ihren spezifischen Zweck angefertigt. 1919 begann die Arbeit an dem ersten Element, das den rein dokumentarischen Charakter des Films veränderte: Eine Schauspielerin verkörpert in der Eingangssequenz von «Krüppelnot und Krüppelhilfe» die berufstätige Mutter eines Kindes, das an Rachitis leidet. «Stundenlang alleine», klagt die Texttafel, nachdem die Arbeiterin ihre karge Dachstube mit dem Kinderbettchen verlässt. Hilfe naht, als sie ihren Sohn in einer städtischen Krüppelfürsorgestelle in Berlin SO36 vorstellt ${ }^{96}$.

Spielfilmelemente mit professionellen Darstellern in medizinische Lehrfilme zu integrieren, war eine Spezialität des Ufa-Kulturfilms und Ausdruck der Maxime, dass nur die dramatische Darstellung auf Dauer belehrende Wirkung entfalte. Die Inszenierung stammt von Curt Thomalla (1890-1939), der noch zu Zeiten des Bild- und Filmamtes mit dem Aufbau eines medizinischen Filmarchivs betraut und in den ersten Tagen der Ufa in deren

94 Niederschrift zur Verhandlung über den Bildstreifen «Krüppelnot und Krüppelhilfe» der Film-Oberprüfstelle vom 28.1.1921. Archiv des Deutschen Filminstituts Frankfurt a. M., Bestand: Zensur, Filmoberprüfstelle B.6.21. Online einsehbar über: http://www.deutschesfilminstitut.de/filme/f035280.htm.

95 Schreiben Konrad Biesalski an Julius Gebauer vom 8. Juli 1914. Archiv Oskar-Helene-Heim, Akte Prof. Dr. Biesalski.

96 Der postalische Bezirk SO36 bildete den proletarisch geprägten Teil des zwischen 1920 und 2001 existierenden Berliner Stadtbezirks Kreuzberg. 
Kulturfilmabteilung übernommen wurde ${ }^{97}$. Insbesondere um die Sexualaufklärungsfilme der Ufa-Kulturabteilung machte sich Thomalla verdient ${ }^{98}$, sie wurden - leicht verschämt - von den Verantwortlichen «Steinachfilme» getauft, nach dem österreichischen Physiologen und Hormonforscher Eugen Steinach (1861-1944) ${ }^{99}$. Curt Thomalla steht wie kaum ein anderer für eine Kontinuität, die vom frühen medizinischen Filmarchiv der Ufa aus den letzten Tagen des Ersten Weltkrieges bis in den Machtapparat der Nationalsozialisten reicht. 1923 schied er bei der Ufa aus und wurde Mitarbeiter des Reichsausschusses für Hygienische Volksbelehrung, parallel dazu entstanden weitere Filmprojekte. Seit Mai 1933 Oberregierungsrat im Ministerium für Volksaufklärung und Propaganda, war er für eine Kampagne zum «Gesetz zur Verhütung erbkranken Nachwuchses〉 zuständig, nach dessen Bestimmungen bis zum Ende des Zweiten Weltkrieges 400000 kranke und behinderte Menschen zwangsweise sterilisiert wurden. In einer Auflage von 30 Millionen Exemplaren soll seine Broschüre «Gesunde Eltern/Gesunde Kinder» erschienen $\operatorname{sein}^{100}$, die als Postwurfsendung an alle Haushalte ging. Der unverfängliche Titel warb für Zwangssterilisierungen ${ }^{101}$.

Thomallas Inszenierung für «Krüppelnot und Krüppelhilfe» war ursprünglich als eigenständige Produktion geplant. Im Ufa-Katalog medizinischer Lehrfilme von 1919 ist das Projekt als «noch in Arbeit befindlich» gekennzeichnet ${ }^{102}$. In dem 1920 uraufgeführten wie in dem heute erhaltenen Film «Krüppelnot und Krüppelhilfe» bildet die oben beschriebene Episode den ersten Akt. Die weiteren vier Akte werden von einer zweiten Spielhandlung gerahmt: Ein gehbehinderter Streichholzverkäufer preist seine Ware vor einer Berliner Litfasssäule an. In dieser Strassenszene werden zufällige Passanten und Käufer miteinbezogen. Dann tritt ein auf Krücken gestützter Leierkastenmann auf. Die beiden Männer werden dem Publikum als Almosenempfänger vorgestellt, die mangels adäquater Therapie und Ausbildung keinem geregelten Broterwerb nachgingen. In der folgenden Einstellung wird ein etwa 10jähriges, gehbehindertes Mädchen gezeigt, das von einem älteren, gebeugten Paar in die Orthopädische Klinik begleitet wird. Auf dem Weg dorthin, das Anstaltsgebäude ist bereits in Sicht, passie-

97 Lebenslauf Curt Thomalla, Bundesarchiv Berlin, Bestand RSK I 574, Blatt 684.

98 Thomalla 1926.

99 Kalbus 1924b.

100 Dies behauptet Thomalla in einem Schreiben an die Reichsschrifttumskammer. Lebenslauf Curt Thomalla, Bundesarchiv Berlin, Bestand RSK I 574, Blatt 684 verso.

101 NS-Volkswohlfahrt 1934.

102 Hier wird «Prof. Dr. Biesalski, Dir. des Oscar-Helene-Heims» als Wissenschaftlicher Bearbeiter und «Dr. Thomalla» als Aufnahmeleiter genannt. Vgl.: Verzeichnis der fertigen und in Arbeit befindlichen Lehrfilme 1919. 
ren Eltern und Tochter den Leierkastenmann. In der Klinik wird das Kind untersucht. Eine Texttafel verkündet: «Diagnose Kinderlähmung». Im Anschluss an die Klinikaufnahme folgt ein Zusammenschnitt der Szenen, die in den zehn Jahren zuvor von Biesalski produziert worden waren: Aufnahmen aus Chirurgie, Turnsaal, Massageabteilung und Lichttherapie, exerzierende armamputierte Soldaten, Lehrwerkstätten und die zahlreichen beschriebenen «fröhlichen Szenen» aus dem Alltag der Anstalt. Erst ganz zum Schluss, am Ende des letzten Aktes, tritt das Mädchen erneut auf. Flankiert von der Texteinblendung «Geheilt entlassen» holt das ältere Paar sie zurück nach Hause. Dem Zuschauer wird so der Eindruck vermittelt, er erlebe das OskarHelene-Heim aus der Perspektive der zehnjährigen Patientin.

Diese mit Laiendarstellern realisierte Rahmenhandlung ist dem Regisseur Nicholas Kaufmann zuzuschreiben ${ }^{103}$. Kaufmann hatte in Berlin Medizin studiert und war offiziell bis 1922 als Internist an der II. Medizinischen Klinik der Charité angestellt ${ }^{104}$. Während dieser Zeit betreute er gemeinsam mit Thomalla das Medizinische Filmarchiv der Ufa. Ein wesentlicher Baustein für den Erfolg Nicholas Kaufmanns bestand im routinierten Einsatz wissenschaftlicher Apparaturen für die filmische Darstellung. Mikroskop und Röntgenstrahlen nutzte er ebenso wie Hochgeschwindigkeitskameras, Endoskope und Gase unterschiedlicher Dichte, deren charakteristische Lichtbrechung er für Trickeffekte einsetzte. Seine Filmographie beginnt 1919 mit «Krüppelnot und Krüppelhilfe». 1927 wird der gebürtige Berliner schweizerischer und US-amerikanischer Nationalität Leiter der Ufa-Kulturfilmabteilung ${ }^{105}$. Er behält dieses Amt mit kurzen Unterbrechungen bis 1944. Kurz vor Ende des Zweiten Weltkrieges geht er in die Schweiz ${ }^{106} .1954$ steht er in der Bundesrepublik erneut in staatlichen Diensten und gibt die Filmkataloge der Bonner Bundeszentrale für gesundheitliche Aufklärung heraus ${ }^{107}$.

«Krüppelnot und Krüppelhilfe» verdeutlicht durch die an ihm vorgenommenen Umarbeitungen die einzelnen Schritte der Entstehung des neuen Genres Kulturfilm. Zunächst hatte der Orthopäde Biesalski selbst die erstmals 1911 gezeigten Bilder überarbeitet. Von der ersten Version der Szene, in der seine Patienten an der Kamera vorbeiziehen, sind nur wenige auf

103 Im Vorspann des Films heisst es «Aus dem Oskar-Helene-Heim, Direktor Prof. Dr. Biesalski. Für die Ufa-Kulturfilmabteilung bearbeitet von Dr. med. Kaufmann». Aufgrund dieser Texttafel wurde der gesamte Film Kaufmann zugeschrieben.

104 Reichshandbuch der deutschen Gesellschaft 1930, 894.

105 NSDAP Gaukartei, Mitgliedsnummer 2826472. Bundesarchiv Berlin, Bestand ehemals Berlin Document Center.

106 Keitz,Ursula von, Nicholas Kaufmann.http://www.deutsches-filminstitut.de/dframe12.htm. 21. Februar 2008

107 Kaufmann 1954. 
Barytpapier abgezogene Standbilder erhalten. Sie zeigen aufrecht schreitende Kinder in einer geordneten Reihe auf einem Berliner Bürgersteig. Schon 1914 perfektionierte er dann die Sequenz, indem er durch den Wechsel der Geschwindigkeiten (ruhige Schritte auf dem Gang in der Anstalt, Versammlung auf der Rampe und das Rennen hinunter in den Wald) die dynamischen Mittel des Films gekonnt einsetzte. Erst der von den UfaRegisseuren veranlasste Einsatz von Laiendarstellern und professionellen Schauspielern verwandelte den Dokumentarfilm des Anstaltsleiters schliesslich in einen Kulturfilm.

Die beiden Kulturfilmer Nicholas Kaufmann und Curt Thomalla begannen ihre Karriere gegen Ende des Ersten Weltkrieges als Mediziner. Sie betrachteten sich selbst jedoch schon bald nach Beginn ihrer Arbeit bei der Ufa vor allem als Regisseure und als Vermittler zwischen den vermeintlichen Bedürfnissen des breiten Publikums und den Vorstellungen ihrer Auftraggeber. Zumindest in den ersten Jahren waren das Ärzte, Krankenhausdirektoren und Behörden. Doch diese Klientel brach in der Zeit der Hyperinflation weg. Der Staat zog sich weitgehend aus dem Filmgeschäft zurück. Kaufmann und Thomalla wandten sich bald jenen Kulturfilmbereichen zu, die Geld einspielten. Bergfilme, Tierfilme und Unterwasseraufnahmen wurden ihre Spezialität ${ }^{108}$. Medizinfilme wie «Krüppelnot und Krüppelhilfe» spielten in Rückblicken auf die Kulturfilmproduktion der späten zwanziger Jahre eine stetig abnehmende Rolle.

Heute prägen Tierfilme und Gebirgsaufnahmen die Vorstellung vom Kulturfilm der zwanziger Jahre weit mehr als die frühen Produktionen. Die Entstehungsbedingungen der frühen Medizinfilme jedoch - das undurchsichtige Anerkennungsverfahren, die steuerliche Förderung und vor allem die Staatsnähe - sind Aspekte der Frühphase, die den «Kulturfilm» weit über das Ende der Weimarer Republik hinaus bestimmten.

\section{Fazit}

Dieser Beitrag befasste sich mit der Entstehung des Genres «deutscher Kulturfilm». Ausgehend von der durch ablehnende Kritik geprägten Situation medizinischer Filme in den ersten anderthalb Jahrzehnten der Filmgeschichte wurde der Übergang vom Kaiserreich in die Weimarer Republik untersucht. Im letzten Jahr des Ersten Weltkrieges entwarf das Bild- und 2008. 
Filmamt der Obersten Heeresleitung, angeregt durch die Thesen des deutschamerikanischen Psychotechnikers Hugo Münsterberg, eine Mischform aus Dokumentar- und Spielfilm. Nur wenige Produktionen aus dieser Zeit wurden abgeschlossen. Zum Tragen kam das neue Konzept zur Generierung von Propagandafilmen erst in Filmen medizinischen Inhalts, die in der unmittelbaren Nachkriegszeit zum Zweck der hygienischen Volksbelehrung in die Kinos gelangten.

Aktenbestände aus dem Bundesarchiv belegen, dass sich die Reichskanzlei bemühte, die 1919 als unrechtmässig erkannte Verstaatlichung der deutschen Filmindustrie für den Bereich der dokumentarischen Filme de facto aufrechtzuerhalten. Es konnte gezeigt werden, dass dies, vor allem bei Hygiene- und Medizinfilmen, weitgehend gelang. Mit Eingriffen in das Steuersystem, neuen Antragsverfahren, einem Kulturfilmausschuss und der Schaffung eines behördlichen Zertifikates, das «Kulturfilmschein» genannt wurde, konnte der Staat weiterhin massgeblichen Einfluss auf die Produktion von Dokumentarfilmen nehmen. Angestellte des ehemaligen Bild- und Filmamtes der Obersten Heeresleitung, wie der Mediziner Curt Thomalla, wechselten in private Gesellschaften. Sie blieben Ansprechpartner für die Behörden und gewährleisteten Kontinuität.

Die enge Kooperation zwischen Staat, Filmindustrie und Interessenverbänden zeigt sich exemplarisch in dem Film «Krüppelnot und Krüppelhilfe». Noch zur Kaiserzeit bemühte sich die preussische Regierung, die Kosten für Behandlung und Ausbildung körperbehinderter Menschen den Kommunen anzulasten. Die sogenannte «Krüppelfürsorge» sollte Bestandteil des Armenrechts und nicht Gegenstand der Sozialversicherungen werden. Parlamentarische Initiativen mit diesem Ziel waren bereits ab 1911 vom preussischen Kultusministerium propagandistisch begleitet worden. 1919 und 1920 wurden in der Vorkriegszeit gefertigte Filmaufnahmen nach den neuerworbenen Kenntnissen über die Beeinflussbarkeit des Publikums durch Spielhandlungen ergänzt. So kam im September 1920 ein Film zur Uraufführung, der ein Projekt der Kaiserzeit mit Mitteln verfolgte, die gegen Ende des Krieges zu Propagandazwecken erarbeitet worden waren.

Der «deutsche Kulturfilm» transportierte die Propagandakonzepte des Ersten Weltkrieges in die Zeit der Weimarer Republik. Filme medizinischen Inhalts blieben die zwanziger Jahre hindurch Instrumente der «hygienischen Volksbelehrung». Ein von der Meinungsvielfalt unterschiedlicher Ideen getragenes Filmwesen konnte sich in Deutschland auf diesem Sektor nicht entwickeln. Pluralismus blieb ausschliesslich künstlerischen Produktionen vorbehalten. Ein Mann, der vom Konzept des «deutschen Kulturfilms» wenig überzeugt war, musste sie sich alle ansehen: der Schriftsteller Carl Bulcke 
(1875-1936). Als Vorsitzender der Berliner Film-Oberprüfstelle war er der oberste Zensor der Weimarer Republik. Er definierte den «Kulturfilm» wie folgt:

Was unter dieser Überschrift läuft, ist etwas sehr Anständiges, Solides, Fleißiges, meist freilich auch stark Langweiliges. Doch weil die gute Absicht überwiegt, soll man von dem sogenannten Kulturfilm nur Gutes und Erfreuliches sagen. Er ist so eine Art von erweitertem Konfirmandenunterricht, zu dem auch Erwachsene Zutritt haben. ${ }^{109}$

\section{Quellen}

\section{Archivalien}

Bundesarchiv, Berlin:

Akten betreffend Film-Angelegenheiten. Vom 27. Mai 1919 bis zum 20. Oktober 1920. Bestand R43 I, 2497.

Auszug aus dem Protokoll der Sitzung des Reichsministeriums vom 27. Mai 1919. Akten betreffend Film-Angelegenheiten, Alte Reichskanzlei. Bundesarchiv Berlin, Bestand R 43 I/ 2497, Blatt 4.

Nachlass Oskar Messter. Preisverzeichnis sämtlicher optischer, mechanischer und chirurgischer Instrumente. Bestand N 1275, Band 234, 235 und 238.

NSDAP Gaukartei, Bestand ehemals Berlin Document Center.

Bestand RSK I 574.

Bundesarchiv-Filmarchiv, Berlin:

Krüppelnot und Krüppelhilfe. Deutschland 1920. Produktion Ufa Kulturabteilung. 35 mm, s/w, 1.062 m. Regie: Nicholas Kaufmann; Kurt Thomalla und Konrad Biesalski.

Geheimes Staatsarchiv Preußischer Kulturbesitz, Berlin:

Rep. 76 VIII B, Nr. 1675 und Nr. 1677.

Archiv Oskar-Helene-Heim, Berlin:

Schreiben Konrad Biesalski an Julius Gebauer vom 8. Juli 1914. Akte Prof. Dr. Biesalski, unpaginierter Bestand.

Deutsches Filminstitut (Onlinearchiv):

Bestand: Zensur, Filmprüfstelle Berlin; http://www.deutsches-filminstitut.de/filme/f035280.htm am 11. August 2008.

\section{Artikel aus Tageszeitungen}

Titel und Datum der Artikel aus Tageszeitungen werden der schnelleren Orientierung halber auch in den Fussnoten wiedergegeben.

"A Report from the Department of Peace", The New York Times (October 11,1916) 10

"A. P. Night Wire: Muensterberg Resigns as Harvard Professor", Los Angeles Times (October $15,1914) \mathrm{I}, 1$

A-j., «Lehrfilme zum Krüppelkongreß. Probevorführungen der Ufa», Filmkurier 196 (vom 3. September 1920)

"Col. Roosevelt Makes Attacks on Muensterberg", Christian Science Monitor (October 27,1916) 5

"Declines German Chair. Prof. Hugo Muensterberg Decides to Remain with Harvard", The Washington Post (April 14,1905) 11

109 Bulcke 1924, 16-18. 
"Decorates Münsterberg", New York Times (August 24,1911) 4

«6. Deutscher Kongress für Krüppelfürsorge», Berliner Volkszeitung 416 (vom 3. September 1920)

«Deutscher Kongreß für Krüppelfürsorge», Deutsche Allgemeine Zeitung 432 (vom 4. September 1920)

"From an American Correspondent: The Kaiser's American Agents. The Failure of a Vast Campaign", The Times, Saturday (September 26, 1914) 3

«Krüppelheime und Krüppelschulen. Der zweite Tag des Kongresses», Berliner Morgenpost 229 (vom 3. September 1920)

"Muensterberg Case Said to Be Before Harvard. President and Fellows Hold a Meeting but Give No Official Statement", Christian Science Monitor (October 30,1916) 1

"Muensterberg Dies Addressing Class. Harvard Psychologist Falls Unconscious Before Sixty Radcliff Girls", The New York Times (December 17, 1916) 19

"Münsterberg, Hugo: A Contradiction of the Allegations That the Kaiser Was Cool to Him", The New York Times (December 8,1910) 12

"Münsterberg, Hugo: To the Editor of the New York Times", The New York Times (November 6,1916$) 10$

"Muensterberg Sees Kultur Worldwide", The New York Times (October 27,1916) 9

"Notable Books in Brief Reviews. Professor Muensterberg and Vachel Lindsay in Appreciations of the Art of the Cinema", The New York Times (June 4,1916) BR 234

"Prof. Münsterberg Speaks. Hoboken Audience Is Told of Future of German 'Kultur'”, Christian Science Monitor (October 27, 1916) 5

"Retention of Muensterberg Is Protested. American Rights League Says his Attitudes on War Inconsistent with Duties as Instructor in United States University", Christian Science Monitor (October 21, 1916)

"Suggest That Harvard Oust Muensterberg. Boston Members of American Rights League Petition the University Authorities", The New York Times (October 22,1916) 3

\section{Bibliographie}

Aufsätze ohne Autorenangabe sind nach ihrem Titel aufgeführt. Wo Vornamen von Autoren fehlen oder nur mit einer Initiale abgekürzt sind, konnten vollständige Angaben nicht ermittelt werden.

Ackerknecht, Erwin, «Das Lichtspiel im Dienste der Bildungspflege», Handbuch für Lichtspielreformer (Berlin 1918)

- Lichtspielfragen (Berlin 1928)

Albrecht, A., «Deutsche Ausstellung 〈Das Gas〉 München 1914. Ein Rundgang durch die Ausstellung», Technische Rundschau, Wochenbeilage zum Berliner Tageblatt 20 (1914) 394-396

Arnheim, Rudolf, «Paukerfilme», Die Weltbühne 28 (1932) 185-187

Barkhausen, Hans, Filmpropaganda für Deutschland im Ersten und Zweiten Weltkrieg (Hildesheim 1982)

Biesalski, Konrad, «Eine praktische Verwendung der Röntgen'schen Photographie», Deutsche Medicinische Wochenschrift 22 (1896) 203-204

Bulcke, Carl, «Haben wir schon einen Kulturfilm?», in: E. Beyfuss/A. Kossowsky, Das Kulturfilmbuch (Berlin 1924) 16-18

Degener, Ernst, «Medizinische Kinematographie», in: Alfred Hay, Photographisches Praktikum für Mediziner und Naturwissenschaftler (Wien 1930) 303-335

Dietrich, Eduard, «Der medizinische Ausschuss der Bildstelle», in: Kulturabteilung der Universum-Film A.-G. (Hrsg.), Das Medizinische Filmarchiv bei der Kulturabteilung der UniversumFilm A.-G. (Berlin 1919) 9-11

Dommann, Monika, Durchsicht. Einsicht. Vorsicht. Eine Geschichte der Röntgenstrahlen 18961963 (Zürich 2003)

Fölsing, Albert, Wilhelm Conrad Röntgen. Aufbruch ins Innere der Materie (München 1995) 
Goergen, Jeanpaul, «〈Soldaten Lieder〉 und 〈Zeichnende Hand〉. Propagandafilme von John Heartfield und George Grosz im Auftrag des Auswärtigen Amtes 1917/18», Kintop 3 (1994) 129-142

- «Filmographie»; in: Klaus Kreimeier/Antje Ehmann/Jeanpaul Goergen, Geschichte des dokumentarischen Films in Deutschland, Band 2: Weimarer Republik. 1918-1933 (Stuttgart 2005) 638-662

Gunthert, André, «Klinik des Sehens. Albert Londe, Wegbereiter der medizinischen Fotografie», Fotogeschichte 21 (2000) 27-40

Haase, W., «Begleittext zu: Amputation eines Unterschenkels. Reichsanstalt für Film und Bild in Wissenschaft und Unterricht», Archivfilm 505, Druck vom 26.10.1941. www.iwf.de/iwf/ medien/infothek?Signatur $=\mathrm{B}+505$ (11.8.2008)

Holl, Ute, Kino, Trance und Kybernetik (Berlin 2002)

«Journalliteratur», Fortschritte auf dem Gebiete der Röntgenstrahlen 1 (1897/1898) 32-40

Jung, Uli/Martin Loiperdinger, «Filmographie», in: Uli Jung/Martin Loiperdinger (Hrsg.), Geschichte des dokumentarischen Films in Deutschland, Band 1: Kaiserreich 1895-1918 (Stuttgart 2006) 510-531

- /Wolfgang Mühl-Benninghaus, «Firmengründungen», in: Uli Jung/Martin Loiperdinger (Hrsg.), Geschichte des dokumentarischen Films in Deutschland, Band 1: Kaiserreich 1895-1918 (Stuttgart 2006) 406-416

Kalbus, Oskar, Der Deutsche Lehrfilm in der Wissenschaft und im Unterricht (Berlin 1922)

- «Abriß einer Geschichte der deutschen Lehrfilmbewegung», in: E. Beyfuss/A. Kossowsky, Das Kulturfilmbuch (Berlin 1924a) 1-13

- «Der Steinachfilm», in:E. Beyfuss/A. Kossowsky, Das Kulturfilmbuch (Berlin 1924b) 223-228

Karlsch, Rainer, «Die wirtschaftliche Entwicklung der Messter-Firmen», in: Martin Loiperdinger, Oskar Messter - Filmpionier der Kaiserzeit (Basel 1994) 149-166

Kaufmann, Nicholas, Filmtechnik und Kultur (Stuttgart/Berlin 1931)

- «Der Film im Dienste der Volksgesundheit», in: Deutsches Gesundheitsmuseum, Zentralinstitut für Gesundheitserziehung e.V.(Hrsg.), Der Film im Dienst der Volksgesundheit (KölnMerheim 1954) 5-7

Keitz, Ursula von, «Wissen als Film. Zur Entwicklung des Lehr- und Unterrichtsfilms», in: Klaus Kreimeier/Antje Ehmann/Jeanpaul Goergen, Geschichte des dokumentarischen Films in Deutschland, Band 2: Weimarer Republik. 1918-1933 (Stuttgart 2005) 120-142

- Nicholas Kaufmann. http://www.deutsches-filminstitut.de/dframe12.htm (21.2.2008)

Keller, Phyllis, States of Belonging. German-American Intellectuals and the First World War (Cambridge, MA 1979)

Kittler, Friedrich, Grammophon. Film. Typewriter (Berlin 1986)

Koerber, Martin, «Oskar Messter - Stationen einer Karriere», in: Martin Loiperdinger, Oskar Messter - Filmpionier der Kaiserzeit (Basel 1994) 27-92

Köhne, Paul, Krankenversicherungsgesetz vom 15. Juni 1883/10. April 1892 (Stuttgart 1892)

Krause, Marcus/Nicholas Pethes, «Einleitung: Die Kinematographie des Menschenversuchs», in: Marcus Krause/Nicholas Pethes, Mr. Münsterberg und Dr. Hyde. Zur Filmgeschichte des Menschenexperiments (Bielefeld 2007) 7-32

Lampe, Felix, «Kulturfilm und Filmkultur», in: E. Beyfuss/A. Kossowsky, Das Kulturfilmbuch (Berlin 1924) 19-27

Lentz, «Ministerialdirektor Dietrich zum 70. Geburtstage», Deutsche Medicinische Wochenschrift 56 (1930) 1749-1750

Loiperdinger, Martin, «Ein deutsches Insekt. Die Biene Maja und ihre Abenteuer (1926)», Filmblatt 37 (2008) 65-80

- /Harald Pulch, "The early years of Oskar Messter: establishing film business in Germany", in: Claire Dupré La Tour/André Gaudreault/Roberta Pearson (eds), Cinema at the Turn of the Century (Lausanne 1998) 271-279

Lück, Helmut, «Hugo Münsterberg», in: Historische Kommission bei der Bayerischen Akademie der Wissenschaften (Hrsg.), Neue deutsche Biographie, Bd. 18 (Berlin 1997) 542-543

Messter, Oskar, Mein Weg mit dem Film (Berlin 1936)

Münsterberg, Hugo, Amerika und der Weltkrieg. Ein amerikanisches Kriegstagebuch (Leipzig 1915)

- Die Amerikaner (2 Bände) (Berlin 1914) 
- The Peace and America (New York/London 1915)

- The Photoplay. A Psychological Study (New York/London 1916)

- The War and America (New York, London 1914)

- Tomorrow. Letters to a friend in Germany (New York/London 1916)

Münsterberg, Margaret, Hugo Münsterberg. His Life and Work (New York/London 1922)

NS-Volkswohlfahrt (Hrsg.), Gesunde Eltern - gesunde Kinder! (Berlin ohne Jahr [1934])

Osten, Philipp, Die Modellanstalt. Über den Aufbau einer «modernen Krüppelfürsorge» 1905-1933 (Frankfurt a. M. 2004)

- «Hygieneausstellungen:Zwischen Volksbelehrung und Vergnügungspark», Deutsches Ärzteblatt 102 (2005) 3085-3088

- «Filmdokument 87. Ärzte als Filmregisseure. Ein Ufa-Kulturfilm aus dem Berliner OskarHelene-Heim für die Heilung und Erziehung gebrechlicher Kinder, aufgenommen in den Jahren 1910 bis 1920», Filmblatt 37 (2008) 36-55

Panter, Peter (= Tucholsky, Kurt), «Der Lesefilm», Hygienischer Wegweiser 8 (1927) 211

Reichsausschuß für hygienische Volksbelehrung, «Prof. Dr. Konrad Biesalski †. Hygienischer Wegweiser», Zentralblatt für Technik und Methodik der hygienischen Volksbelehrung 5 (1930) 57

Reichshandbuch der deutschen Gesellschaft, Das Handbuch der Persönlichkeiten in Wort und Bild (Berlin 1930)

Reyer, Jürgen, Alte Eugenik und Wohlfahrtspflege. Entwertung und Funktionalisierung der Fürsorge vom Ende des 19. Jahrhunderts bis zur Gegenwart (Freiburg im Breisgau 1991)

Roesle, E., Sonder-Katalog für die Gruppe Statistik der wissenschaftlichen Abteilung der Internationalen Hygiene-Ausstellung Dresden 1911 (Dresden 1911)

Rothe, A. von, «Operationsfilme», in: E. Beyfuss/A. Kossowsky, Das Kulturfilmbuch (Berlin 1924) 229-234

Saretzki, Thomas, Reichsgesundheitsrat und Preußischer Landesgesundheitsrat in der Weimarer Republik (Berlin 2000)

Schlossmann, Arthur, Die öffentliche Krüppelfürsorge. Das preußische Gesetz vom 6. Mai 1920 nebst den Ausführungsbestimmungen (Berlin 1920)

Schmidt, Ulf, «Der Blick auf den Körper». Sozialhygienische Filme, Sexualaufklärung und Propaganda in der Weimarer Republik», in: Hans-Michael Bock/Wolfgang Jacobsen/Jörg Schöning/Malte Hagener (Hrsg.), Geschlecht in Fesseln. Sexualität zwischen Aufklärung und Ausbeutung im Weimarer Kino (München 2000a) 23-46

- «Sozialhygienische Filme und Propaganda in der Weimarer Republik», in: Dietmar Jazbinsek (Hrsg.), Gesundheitskommunikation (Wiesbaden 2000b) 53-81

Schweisheimer, Waldemar, Die Bedeutung des Films für Soziale Hygiene und Medizin (München 1920)

Seeger, Ernst, «Staat und Kulturfilm», in: E. Beyfuss/A. Kossowsky, Das Kulturfilmbuch (Berlin 1924) $72-75$

Thomalla, Curt, Falsche Scham (Berlin 1926)

«Verzeichnis der fertigen und in Arbeit befindlichen Lehrfilme des «Medizinischen Filmarchives»», in: Kulturabteilung der Universum-Film A.-G. (Hrsg.), Das medizinische Filmarchiv bei der Kulturabteilung der Universum-Film A.-G (Berlin 1919) 44-56

Vöhringer, Margarete, Avantgarde und Psychotechnik. Wissenschaft, Kunst und Technik der Wahrnehmungsexperimente in der frühen Sowjetunion (Göttingen 2007)

Vorstand des Vereins zur Veranstaltung der Internationalen Hygiene-Ausstellung Dresden 1911 e.V. (Hrsg.): Ausstellungsbedingungen für die Wissenschaftliche Abteilung (Dresden 1909) 2

Wendenburg, Friedrich, Soziale Hygiene (= Handbücherei für Staatsmedizin 13) (Berlin 1929)

«Zum Zwecke der Propaganda», Zeitschrift für Krüppelfürsorge 5 (1912) 5-11 